MITSUBISHI ELECTRIC RESEARCH LABORATORIES

http://www.merl.com

\title{
Cognitive Single Carrier Systems: Joint Impact of Multiple Licensed Transceivers
}

\author{
Kim, K.J.; Wang, L.; Duong, T.Q.; Elkashlan, M.; Poor, H.V. \\ TR2014-089 December 2014
}

\begin{abstract}
In this paper, the impact of interference from multiple licensed transceivers on cognitive underlay single-carrier systems is examined. Specifically, the situation is considered in which the secondary network is limited by three key parameters: 1) maximum transmit power at the secondary transmitter, 2) peak interference power at the primary receivers, and 3) interference power from the primary transmitters. For this cognitive underlay single-carrier system, the signal-to-interference ratio (SIR) of the secondary network is obtained for transmission over frequency-selective fading channels. Based on this, a new closed-form expression for the cumulative distribution function of the SIR is evaluated, from which the outage probability and the ergodic capacity are derived. Further insights are established by analyzing the asymptotic outage probability and the asymptotic ergodic capacity in the high-transmissionpower regime. In particular, it is corroborated that the asymptotic outage diversity gain is equal to the multipath gain of the frequency-selective channel in the secondary network. The asymptotic ergodic capacity also gives new insight into the additional power cost for different network parameters while maintaining a specified target ergodic capacity. Illustrative numerical examples are presented to validate the outage probability and ergodic capacity under different interference power profiles.
\end{abstract}

IEEE Transactions on Wireless Communications

This work may not be copied or reproduced in whole or in part for any commercial purpose. Permission to copy in whole or in part without payment of fee is granted for nonprofit educational and research purposes provided that all such whole or partial copies include the following: a notice that such copying is by permission of Mitsubishi Electric Research Laboratories, Inc.; an acknowledgment of the authors and individual contributions to the work; and all applicable portions of the copyright notice. Copying, reproduction, or republishing for any other purpose shall require a license with payment of fee to Mitsubishi Electric Research Laboratories, Inc. All rights reserved. 



\title{
Cognitive Single Carrier Systems: Joint Impact of Multiple Licensed Transceivers
}

\author{
Kyeong Jin Kim, Senior Member, IEEE, Lifeng Wang, Student Member, IEEE, \\ Trung Q. Duong, Senior Member, IEEE, Maged Elkashlan, Member, IEEE, and \\ H. Vincent Poor Fellow, IEEE
}

\begin{abstract}
In this paper, the impact of interference from multiple licensed transceivers on cognitive underlay single carrier systems is examined. Specifically, the situation is considered in which the secondary network is limited by three key parameters: 1) maximum transmit power at the secondary transmitter, 2) peak interference power at the primary receivers, and 3) interference power from the primary transmitters. For this cognitive underlay single carrier system, the signal-to-interference ratio (SIR) of the secondary network is obtained for transmission over frequency selective fading channels. Based on this, a new closedform expression for the cumulative distribution function of the SIR is evaluated, from which the outage probability and the ergodic capacity are derived. Further insights are established by analyzing the asymptotic outage probability and the asymptotic ergodic capacity in the high transmission power regime. In particular, it is corroborated that the asymptotic outage diversity gain is equal to the multipath gain of the frequency selective channel in the secondary network. The asymptotic ergodic capacity also gives new insight into the additional power cost for different network parameters while maintaining a specified target ergodic capacity. Illustrative numerical examples are presented to validate the outage probability and ergodic capacity under different interference power profiles.
\end{abstract}

Index Terms-Cognitive radio, ergodic capacity, frequency selective fading, outage probability, primary transceivers, single carrier transmission.

\section{INTRODUCTION}

$\mathbf{R}$ ADIO frequency spectrum is an increasingly scarce and expensive wireless resource due to the upsurge in demand for multimedia services in current and future generation wireless networks. Unfortunately, recent measurement campaigns have found that the radio frequency spectrum is not being efficiently utilized [1]-[7]. Cognitive radio, proposed by Mitola in [8], has the potential to mitigate such inefficiency. Particularly, by allowing a secondary user (SU) to reuse the radio spectrum that is licensed to a primary user (PU), the scarcity of frequency spectrum can be alleviated. Several

Manuscript received November 27, 2013; revised March 18, 2014; accepted May 7, 2014. The editor coordinating the review of this paper and approving it for publication was Prof. Y. Sanada.

K. J. Kim is with Mitsubishi Electric Research Laboratories (MERL), Cambridge, MA, USA. Part of this work was completed while he was in Inha University, Korea.

L. Wang and M. Elkashlan are with Queen Mary University of London, London, UK.

T. Q. Duong is with Queen's University Belfast, Belfast, UK.

H. V. Poor is with the Department of Electrical Engineering, Princeton University, Princeton, NJ, USA.

This research was supported in part by the U. S. National Science Foundation under Grant ECCS-1343210. approaches to cognitive radio such as overlay, interweave, and underlay spectrum sharing have been considered [9]. Among them, the most promising approach is underlay spectrum sharing in which the SU simultaneously transmits in the same radio spectrum as the PU, provided that the secondary transmission does not exceed the maximum interference constraint set by the primary network [10]. One of the drawbacks of underlay spectrum sharing is the need to limit the transmit power of the SU transmitter (SU-Tx) to avoid any deleterious effect on the PU receiver (PU-Rx). In some practical scenarios, the cognitive radio network may not be feasible due to heavy pathloss and severe shadowing [11]. As such, several advanced transmission technologies have been introduced to enhance the performance of underlay spectrum sharing such as cognitive relaying [12] and cognitive multiuser diversity [13].

Cognitive underlay spectrum sharing with single carrier transmission was first considered in [14]. Since then, promising strategies for cognitive underlay single carrier systems have been proposed such as cognitive relay selection [15] and cognitive decode-and-forward (DF) with selection combining [16]. Indeed, cognitive underlay spectrum sharing has been proven to boost the outage probability, ergodic capacity, and average symbol error rate (ASER) of single carrier systems relative to non-spectrum sharing single carrier systems [17][20]. In [14]-[16], only a single PU-Rx is considered, while multiple PU-Rxs are considered in non-single carrier transmission in [21]. Furthermore, most previous works assume that the PU transmitter (PU-Tx) is located far enough away so as not to impinge any significant interference on the SU receiver (SU-Rx) [22]. As such, only limited studies have taken into account the interference from either a single or multiple PUTxs (e.g., [14], [21], [23]).

In this paper, in contrast to [14]-[25], we focus on the co-existence of multiple PU-Rxs and multiple interfering PUTxs in cognitive underlay single carrier systems. We consider the practical case of non-identical frequency selective fading between all the PUs and the SUs. ${ }^{1}$ Thus, compared with existing works in [13-15], [20], and [21], the impact of multiple licensed primary transceivers on the performance of spectrum sharing single carrier systems in frequency selective fading channels is first investigated in this paper under a joint constraint on peak interference power at the primary receiver and maximum transmit power at the secondary user. Specifi-

\footnotetext{
${ }^{1}$ For non-spectrum sharing systems, the effects of cochannel interference (CCI) have been examined under identical frequency selective fading in [20]
} and non-identical Nakagami- $m$ fading in [26]. 
cally, the novel contributions of this paper are summarized as follows:

- We consider the joint impact of multiple PU-Txs and multiple PU-Rxs on the performance of cognitive underlay single carrier systems. Motivated by [15] and [21], we employ three interrelated power constraints, namely, the interference power from the PU-Txs on the SU-Rx, denoted by $P_{I_{p}}$, the peak interference power from the SU-Tx on the PU-Rxs, denoted by $I_{p}$, and the maximum transmit power at the SU-Tx, denoted by $P_{T}$.

- We characterize the end-to-end signal-to-interference plus noise ratio (SINR) at the SU-Rx in terms of the channel impulse responses of the frequency selective fading channels. Using this statistic, new analytical expressions for the outage probability and the ergodic capacity are obtained. These results are used to evaluate the impact of the multiple PU-Txs and PU-Rxs on the secondary network.

- We derive the asymptotic outage probability in the high transmission power regime. This reveals additional insights into the diversity gain, which is equal to the multipath gain of the frequency selective channel in the secondary network. Interestingly, we confirm that the diversity gain is lost when the interference power from the PU-Txs is proportional to the maximum transmit power at the SU-Tx.

- We derive the asymptotic ergodic capacity in the high transmission power regime under the proportional interference power constraint. Using this new result, we confirm that the multiplexing gain is 1 . Moreover, given a specified target ergodic capacity, we accurately calculate the additional power cost for different network parameters.

Notation: The superscript $(\cdot)^{H}$ denotes complex conjugate transposition; $\boldsymbol{I}_{N}$ is an $N \times N$ identity matrix; $\mathbf{0}$ denotes an all-zeros matrix of appropriate dimensions; $\mathcal{C N}\left(\mu, \sigma^{2}\right)$ denotes the complex Gaussian distribution with mean $\mu$ and variance $\sigma^{2} ; \mathbb{C}^{m \times n}$ denotes the vector space of all $m \times n$ complex matrices; $F_{\varphi}(\cdot)$ denotes the cumulative distribution function (CDF) of the random variable (RV) $\varphi ; E_{a}\{\cdot\}$ denotes expectation with respect to $a$. The probability density function (PDF) of $\varphi$ is denoted by $f_{\varphi}(\cdot)$.

The rest of the paper is organized as follows. In Section II, we first detail the system and channel model of the proposed cognitive underlay single carrier systems. In Section III, the SINR and signal-to-interference ratio (SIR) are defined, based on which the outage probability and ergodic capacity are derived. Simulation results are presented in Section IV, and conclusions are drawn in Section V.

\section{SyStem AND CHANNEL MODEL}

We assume a single antenna equipped cognitive underlay single carrier system with $M$ PU-Txs transmitting to $N$ PU-Rxs in the primary network. In the secondary network, we consider that the SU-Tx transmits to the SU-Rx in the same primary licensed frequency band subject to interference constraints imposed by the PU-Rxs. Unlike [12], [22], [24],

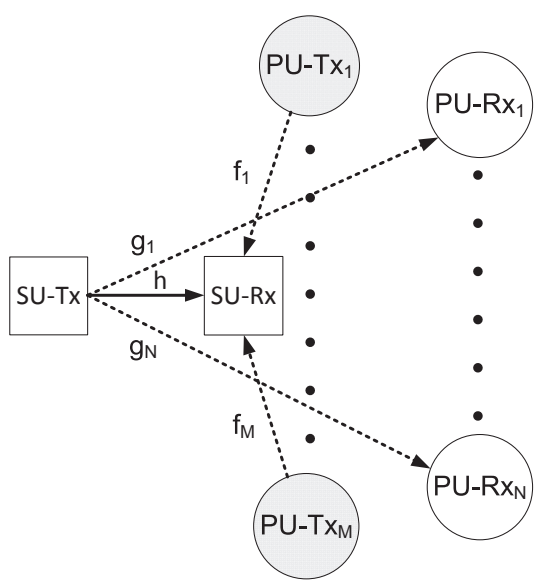

Fig. 1. Illustration of the spectrum sharing single carrier network with multiple PU-Rxs and multiple PU-Txs.

and [25] which ignored interference from the PU-Txs, we consider that all PU-Txs impinge significant interference upon the received signals at the SU-Rx. The set of instantaneous impulse channel responses in the system are detailed as follows:

- The frequency selective channel from the SU-Tx to the SU-Rx is defined by a channel vector $\boldsymbol{h} \triangleq\left[h_{0}, \ldots, h_{L_{1}-1}\right]^{T} \in \mathbb{C}^{L_{1} \times 1}$. The path loss and the multipath channel length of $\boldsymbol{h}$ are denoted by $\alpha_{h}$ and $L_{1}$, respectively.

- The frequency selective interfering channel from the $k$ th $\mathrm{PU}^{-\mathrm{Tx}_{k}}$ to the SU-Rx is defined by a channel vector $\boldsymbol{f}_{k} \triangleq\left[f_{k, 0}, \ldots, f_{k, L_{2}-1}\right]^{T} \in \mathbb{C}^{L_{2} \times 1}$. The path loss over the channel $\boldsymbol{f}_{k}$ and the multipath channel length of all $\left\{\boldsymbol{f}_{k}\right\}_{k=1}^{M}$ are denoted by $\alpha_{f, k}$ and $L_{2}$, respectively.

- The frequency selective interfering channel from the SU-Tx to the $k$ th $\mathrm{PU}^{-\mathrm{Rx}_{k}}$ is defined by $\boldsymbol{g}_{k} \triangleq\left[g_{k, 0}, \ldots, g_{k, L_{3}-1}\right]^{T} \in \mathbb{C}^{L_{3} \times 1}$. The path loss over the channel $\boldsymbol{g}_{k}$ and the multipath channel length of all $\left\{\boldsymbol{g}_{k}\right\}_{k=1}^{N}$ are denoted by $\alpha_{g, k}$ and $L_{3}$, respectively.

Recall that we denote the maximum transmit power at the SU-Tx by $P_{T}$ and the peak allowable interference at all the PU-Rxs by $I_{p}$. Given these two distinctive constraints, the transmit power at the SU-Tx is defined as [15], [27]

$$
P_{s}=\min \left(P_{T}, \frac{I_{p}}{\max _{k=1, \cdots, N}\left\{\alpha_{g, k}\left\|\boldsymbol{g}_{k}\right\|^{2}\right\}}\right) .
$$

Binary phase shift keying (BPSK) modulation is applied such that $Q$ modulated data symbols transmitted by the PU-Tx form a transmit symbol block $\boldsymbol{x} \in \mathbb{C}^{Q \times 1} \in\{-1,1\}^{Q}$ satisfying $E_{\boldsymbol{x}}\{\boldsymbol{x}\}=\mathbf{0}$ and $E_{\boldsymbol{x}}\left\{\boldsymbol{x} \boldsymbol{x}^{H}\right\}=\boldsymbol{I}_{Q}$. A cyclic prefix (CP) comprising of $Q_{g}$ symbols is appended to the front of $\boldsymbol{x}$ to prevent inter-block symbol interference (IBSI) [28], [29]. After removing the signal associated with the $\mathrm{CP}$, the received signal at the SU-Rx can be written as

$$
\boldsymbol{y}=\sqrt{P_{s} \alpha_{h}} \boldsymbol{H} \boldsymbol{x}+\sum_{p=1}^{M} \sqrt{P_{I_{p}} \alpha_{f, p}} \boldsymbol{F}_{p} \tilde{\boldsymbol{x}}_{p}+\boldsymbol{z}
$$


where $P_{I_{p}}$ is the interference power from the $p$ th PU-Tx, $\boldsymbol{H} \in$ $\mathbb{C}^{Q \times Q}$ is the right circulant channel matrix [14], [30] specified by $\boldsymbol{h},\left\{\boldsymbol{F}_{p}\right\}_{p=1}^{K} \in \mathbb{C}^{Q \times Q}$ is the set of right circulant channel matrices specified by $\left\{\boldsymbol{f}_{p}\right\}_{p=1}^{K}$, and $\boldsymbol{z} \sim \mathcal{C N}\left(\mathbf{0}, \sigma_{n}^{2} \boldsymbol{I}_{Q}\right)$ denotes additive white Gaussian noise at the SU-Rx. We define the interfering transmit symbol from the PU-Tx as $\tilde{\boldsymbol{x}}_{p} \in \mathbb{C}^{Q \times 1}$ which satisfies $E_{\tilde{\boldsymbol{x}}_{p}}\left\{\tilde{\boldsymbol{x}}_{p}\right\}=\mathbf{0}$ and $E_{\tilde{\boldsymbol{x}}_{p}}\left\{\tilde{\boldsymbol{x}}_{p} \tilde{\boldsymbol{x}}_{p}^{H}\right\}=\boldsymbol{I}_{Q}$. Since, in general, $\max \left\{L_{1}, L_{2}, L_{3}\right\}$ is smaller than the block size $Q$, a necessary zero padding is required for $\boldsymbol{h},\left\{\boldsymbol{f}_{p}\right\}$, and $\left\{\boldsymbol{g}_{k}\right\}$ to form a $Q \times Q$ circulant channel matrix.

To determine the effects of the PU-Tx interference on the secondary network, we shall first define the instantaneous SINR at the SU-Rx as follows.

Definition 1: Applying the properties of the right circulant channel matrix ${ }^{2}$ [14], [30] and the frequency selective channel models given above, the instantaneous SINR is defined as

$$
\begin{aligned}
\gamma_{\mathrm{SINR}} & =\frac{\min \left(\tilde{P}_{T}, \frac{\tilde{I}_{p}}{\max _{k=1, \cdots, N}\left\{\tilde{\alpha}_{g, k}\left\|\boldsymbol{g}_{k}\right\|^{2}\right\}}\right) \tilde{\alpha}_{h}\|\boldsymbol{h}\|^{2}}{\sum_{p=1}^{M} \tilde{P}_{I_{p}} \tilde{\alpha}_{f, p}\left\|\boldsymbol{f}_{p}\right\|^{2}+1} \\
& \approx \frac{\min \left(\tilde{P}_{T}, \frac{\tilde{I}_{p}}{\max _{k=1, \cdots, N}\left\{\tilde{\alpha}_{g, k}\left\|\boldsymbol{g}_{k}\right\|^{2}\right\}}\right) \tilde{\alpha}_{h}\|\boldsymbol{h}\|^{2}}{\sum_{p=1}^{M} \tilde{P}_{I_{p}} \tilde{\alpha}_{f, p}\left\|\boldsymbol{f}_{p}\right\|^{2}} \\
= & \min \left(\tilde{P}_{T}, \frac{\tilde{I}_{p}}{X}\right) Y \triangleq \gamma
\end{aligned}
$$

where $\gamma$ is the instantaneous SIR normalized by the noise variance. We define $X \triangleq \max _{k=1, \cdots, N}\left\{\tilde{\alpha}_{g, k}\left\|\boldsymbol{g}_{k}\right\|^{2}\right\}$ and $Y \triangleq \frac{A}{B}$ with $A \triangleq \tilde{\alpha}_{h}\|\boldsymbol{h}\|^{2}$, and $B \triangleq \sum_{p=1}^{M} \tilde{P}_{I_{p}} \tilde{\alpha}_{f, p}\left\|\boldsymbol{f}_{p}\right\|^{2}$. In addition, we define the normalized quantities of $\tilde{P}_{T} \triangleq P_{T} / \sigma_{n}^{2}$, $\tilde{I}_{p} \triangleq I_{p} / \sigma_{n}^{2}, \quad \tilde{P}_{I_{p}} \triangleq P_{I_{p}} / \sigma_{n}^{2}, \quad \tilde{\alpha}_{h} \triangleq \alpha_{h} / \sigma_{n}^{2}, \quad \tilde{\alpha}_{g, k} \triangleq \alpha_{g, k} / \sigma_{n}^{2}$, and $\tilde{\alpha}_{f, p} \triangleq \alpha_{f, p} / \sigma_{n}^{2}$.

Note that in the definition of the instantaneous SINR, we assume that the channels are jointly independent and $\boldsymbol{x}$ is independent of $\left\{\tilde{\boldsymbol{x}}_{p}\right\}_{p=1}^{M}$. We also note that the $\operatorname{SIR} \gamma$ is an accurate approximation for the SINR $\gamma_{\text {SINR }}$ in the high interference regime.

\section{Performance Analysis}

In this section, we derive the CDF and PDF of the instantaneous SIR which is a non-trivial problem due to the presence of multiple PU-Txs and PU-Rxs in non-identical frequency selective fading environments. Based on these conditions, we present new closed-form expressions for the outage probability and the ergodic capacity.

\section{A. Distribution of the instantaneous SIR}

The CDF of the instantaneous SIR is defined as $F_{\gamma}(x) \triangleq \operatorname{Pr}\left(\min \left(\tilde{P}_{T}, \frac{\tilde{I}_{p}}{X}\right) Y \leq x\right)$. As such, we can compute

${ }^{2}$ From (2), an instantaneous signal power is defined as $\frac{P_{s} \alpha_{h} \operatorname{Trace}\left(\|\boldsymbol{H}\|^{2}\right)}{Q}$, which is equivalent to $P_{s} \alpha_{h}\|\boldsymbol{h}\|^{2}$ [14], [31]. Similarly, an instantaneous interference plus noise power becomes $\frac{\sum_{p=1}^{M} P_{I_{p}} \alpha_{f, p} \operatorname{Trace}\left(\left\|\boldsymbol{F}_{p}\right\|^{2}\right)+\operatorname{Trace}\left(\|\boldsymbol{z}\|^{2}\right)}{Q}=\sum_{p=1}^{M} P_{I_{p}} \alpha_{f, p}\left\|\boldsymbol{f}_{p}\right\|^{2}+\sigma_{n}^{2}$.
$F_{\gamma}(x)$ as follows

$$
\begin{aligned}
F_{\gamma}(x)= & \operatorname{Pr}\left(Y \leq x / \tilde{P}_{T}, \tilde{I}_{p} \geq X \tilde{P}_{T}\right)+ \\
& \operatorname{Pr}\left(Y \leq X x / \tilde{I}_{p}, \tilde{I}_{p}<X \tilde{P}_{T}\right) \\
= & F_{Y}\left(x / \tilde{P}_{T}\right) F_{X}(\mu)+\underbrace{\int_{\mu}^{\infty} F_{Y}\left(x t / \tilde{I}_{p}\right) f_{X}(t) d t}_{\mathcal{I}_{1}(x)}
\end{aligned}
$$

where $\mu \triangleq \tilde{I}_{p} / \tilde{P}_{T}$ is the ratio of the normalized peak interference at the PU-Rxs relative to the maximum transmit power at the SU-Tx. To compute (4), we need to know the following $\mathrm{CDF}$ and PDF of the RV $X$, which are respectively given by [22], [26]

$$
\begin{aligned}
F_{X}(x)= & 1+\sum_{k=1}^{N} \frac{(-1)^{k}}{k !} \underbrace{\sum_{n_{1}=1}^{N} \cdots \sum_{n_{k}=1}^{N}}_{\left|n_{1} \cup n_{2} \cup \cdots \cup n_{k}\right|=k} \sum_{l_{1}=0}^{L_{3}-1} \cdots \sum_{l_{k}=0}^{L_{3}-1} \\
& \prod_{t=1}^{k}\left(\frac{\left(\beta_{g, n_{t}}\right)^{l_{t}}}{l_{t} !}\right) x^{\sum_{t=1}^{k} l_{t}} e^{-\left(\sum_{t=1}^{k} \beta_{g, n_{t}}\right) x} \mathrm{U}(x) \\
= & 1+\widetilde{\sum}\left[x^{\tilde{l}} e^{-\tilde{\beta}_{g} x} \mathrm{U}(x)\right], \text { and } \\
f_{X}(x)= & \widetilde{\sum}\left[\tilde{l} \tilde{l}^{\tilde{l}-1} e^{-\tilde{\beta}_{g} x} \mathrm{U}(x)\right]-\widetilde{\sum}\left[\tilde{\beta}_{g} x^{\tilde{l}} e^{-\tilde{\beta}_{g} x} \mathrm{U}(x)\right]
\end{aligned}
$$

where $\left|n_{1} \bigcup n_{2} \bigcup \cdots \cup n_{k}\right|$ denotes the cardinality of the union of $k$ indices and $\mathrm{U}(\cdot)$ denotes the unit step function. To simplify notation, we define $\beta_{h} \triangleq \frac{1}{\tilde{\alpha}_{h}}, \beta_{g, k} \triangleq \frac{1}{\tilde{\alpha}_{g, k}}$, and $\beta_{f, p} \triangleq \frac{1}{\tilde{\alpha}_{f, p}}$ in (5). We also define

$$
\begin{aligned}
\widetilde{\Sigma}[.] & \triangleq \sum_{k=1}^{N} \frac{(-1)^{k}}{k !} \underbrace{\sum_{n_{1}=1}^{N} \cdots \sum_{n_{k}=1}^{N} \sum_{l_{1}=0}^{L_{3}-1} \cdots \sum_{l_{k}=0}^{L_{3}-1}}_{\left|n_{1} \cup n_{2} \cup \cdots \cup n_{k}\right|=k} \\
& \prod_{t=1}^{k}\left(\frac{\left(\beta_{g, n_{t}}\right)^{l_{t}}}{l_{t} !}\right)[\cdot], \tilde{l} \triangleq \sum_{t=1}^{k} l_{t}, \text { and } \tilde{\beta}_{g} \triangleq\left(\sum_{t=1}^{k} \beta_{g, n_{t}}\right) .
\end{aligned}
$$

For non-identical Rayleigh fading channels, a similar form of the $\mathrm{CDF}$ is provided in [32]. Note that (5) provides the $\mathrm{CDF}$ and PDF of the RV $X$ for non-identically distributed frequency selective fading channels. Now an additional CDF of the RV $Y$ can be obtained from the following lemma.

Lemma 1: When the channel impulse responses are composed of independent and identically distributed (i.i.d.) complex Gaussian random variables with zero means and unit variances, the $\mathrm{CDF}$ of $Y$ can be written as

$F_{Y}(y)=1-\sum_{l=0}^{L_{1}-1} \sum_{i=1}^{M} \sum_{j=1}^{L_{2}} \Lambda_{i, j, l} y^{l}\left(y+\frac{1}{\beta_{h} \tilde{P}_{I_{i}} \tilde{\alpha}_{f, i}}\right)^{-(l+j)} \mathrm{U}(y)$

where $\quad \Lambda_{i, j, l} \triangleq \frac{(-1)^{j}\left(\beta_{h}\right)^{-j} \theta_{i, j} \Gamma(l+j)}{\Gamma(l+1) \Gamma(j)} \quad$ with $\Gamma(\cdot)$ denoting the gamma function, and $\theta_{i, j} \triangleq \frac{(-1)^{L_{2}}}{\left(\tilde{P}_{I_{i}} \tilde{\alpha}_{f, i}\right)^{L_{2}}} \sum_{S(i, j)} \prod_{k=1, k \neq i}^{M}\left(\begin{array}{c}L_{2}+q_{k}-1 \\ q_{k}\end{array}\right) \frac{\left(\tilde{P}_{I_{k}} \tilde{\alpha}_{f, k}\right)^{q_{k}}}{\left(1-\frac{\tilde{P}_{I_{k}} \tilde{\alpha}_{f, k}}{\tilde{P}_{I_{i}} \tilde{\alpha}_{f, i}}\right)^{L_{2}+q_{k}}}$. 
In addition, $S(i, j)$ denotes a set of $M$-tuples satisfying the following condition:

$$
S(i, j) \triangleq\left\{\left(q_{1}, \ldots, q_{M}\right): \sum_{k=1}^{M} q_{k}=L_{2}-j \text { with } q_{i}=0\right\} .
$$

A.

Proof: A proof of this lemma is provided in Appendix

Based on the CDF of $X$ in (5) and the CDF of $Y$ in (6), we can compute the first part in the right hand side of (4). Next, we proceed to evaluate the integral in the right hand side of (4) denoted as $\mathcal{I}_{1}(x)$. After some manipulations, we arrive at the following form for $\mathcal{I}_{1}(x)$ :

$$
\begin{aligned}
\mathcal{I}_{1}(x)= & 1-F_{X}(\mu)- \\
& \widetilde{\sum}\left[\sum_{l=0}^{L_{1}-1} \sum_{i=1}^{M} \sum_{j=1}^{L_{2}} \Lambda_{i, j, l} \tilde{l}\left(x / \tilde{I}_{p}\right)^{-j} \mathcal{J}_{1}(x)\right]+ \\
& \widetilde{\sum}\left[\sum_{l=0}^{L_{1}-1} \sum_{i=1}^{M} \sum_{j=1}^{L_{2}} \Lambda_{i, j, l} \tilde{\beta}_{g}\left(x / \tilde{I}_{p}\right)^{-j} \mathcal{J}_{2}(x)\right]
\end{aligned}
$$

with

$$
\begin{aligned}
\mathcal{J}_{1}(x)= & e^{-\tilde{\beta}_{g} \mu} \sum_{p=0}^{l+\tilde{l}-1}\left(\begin{array}{c}
l+\tilde{l}-1 \\
p
\end{array}\right) \mu^{l+\tilde{l}-1-p} \\
& \left(\mu+\frac{\tilde{I}_{p}}{\beta_{h} \tilde{P}_{I_{i}} \tilde{\alpha}_{f, i} x}\right)^{p-j-l+1} \Gamma(p+1) \\
& \mathbb{U}\left(p+1, p-j-l+2, \tilde{\beta}_{g}\left(\mu+\frac{\tilde{I}_{p}}{\beta_{h} \tilde{P}_{I_{i}} \tilde{\alpha}_{f, i} x}\right)\right), \text { and } \\
\mathcal{J}_{2}(x)= & e^{-\tilde{\beta}_{g} \mu} \sum_{p=0}^{l+\tilde{l}}\left(\begin{array}{c}
l+\tilde{l} \\
p
\end{array}\right) \mu^{l+\tilde{l}-p} \\
& \left(\mu+\frac{\tilde{I}_{p}}{\beta_{h} \tilde{P}_{I_{i}} \tilde{\alpha}_{f, i} x}\right)^{p-j-l+1} \Gamma(p+1) \\
& \mathbb{U}\left(p+1, p-j-l+2, \tilde{\beta}_{g}\left(\mu+\frac{\tilde{I}_{p}}{\beta_{h} \tilde{P}_{I_{i}} \tilde{\alpha}_{f, i} x}\right)\right)
\end{aligned}
$$

where $\mathbb{U}(\cdot, \cdot ; \cdot)$ denotes the confluent hypergeometric function [33, Eq. 9.211.4]. The derivation of (7) is provided in Appendix B.

Now using (5), (6), and (7), a closed-form expression for the CDF of the SIR is given by (9) at the top of the next page. Based on (9), the outage probability is readily obtained. Next, we proceed to derive new closed-form expressions for the asymptotic outage probability and the ergodic capacity.

\section{B. Asymptotic Outage Probability}

Theorem 1: Let $G_{d}$ be the outage diversity gain defined as

$$
G_{d} \triangleq \lim _{\substack{\tilde{P}_{T} \rightarrow \infty, \tilde{I}_{p} \rightarrow \infty, \mu=\text { const }}} \frac{\log \left(P_{\text {out }}\left(\gamma_{\mathrm{th}}\right)\right)}{\log \left(\gamma_{\mathrm{th}} / \tilde{P}_{T}\right)} .
$$

As such, we find that the achievable diversity gain when $\tilde{I}_{p}$ is proportional to $\tilde{P}_{T}$ is derived as $G_{d}=L_{1}$ which is the multipath gain of the frequency selective channel in the secondary network. However, when $\tilde{P}_{T}$ is fixed and $\tilde{I}_{p}$ is independent of $\tilde{P}_{T}$ (i.e., non-constant ratio of $\mu$ ), the achievable diversity gain diminishes to $G_{d}=0^{3}$.

Proof: A proof of this theorem is provided in Appendix C.

Corollary 1: When the interference powers from the PUTxs are equal or proportional to $\tilde{P}_{T}$, we find that the cognitive underlay single carrier system yields no diversity gain.

Proof: Without loss of generality, we assume that $\tilde{P}_{I_{k}}=$ $\tilde{P}_{I}$, for $k=1,2, \ldots, M$. From (C.5), we can rewrite the asymptotic outage probability as follows:

$$
P_{\text {out }}\left(\gamma_{\text {th }}\right) \underset{\substack{\tilde{P}_{T} \rightarrow \infty, \tilde{T}_{p} \rightarrow \infty, \mu=\text { const }}}{\approx} \tilde{\Omega}_{1}\left(\frac{\tilde{P}_{I} \gamma_{\text {th }}}{\tilde{P}_{T}}\right)^{L_{1}}
$$

where

$$
\begin{aligned}
\tilde{\Omega}_{1}= & \left(F_{X}(\mu)+(1 / \mu)^{L_{1}} \widetilde{\sum}\left[\tilde{l}\left(\tilde{\beta}_{g}\right)^{-\left(L_{1}+\tilde{l}\right)} \Gamma\left(L_{1}+\tilde{l}, \mu \tilde{\beta}_{g}\right)-\right.\right. \\
& \left.\left.\left(\tilde{\beta}_{g}\right)^{-\left(L_{1}+\tilde{l}\right)} \Gamma\left(L_{1}+\tilde{l}+1, \mu \tilde{\beta}_{g}\right)\right]\right) \\
& \sum_{i=1}^{M} \sum_{j=1}^{L_{2}} \frac{(-1)^{j} \theta_{i, j}\left(\beta_{h}\right)^{L_{1}} \Gamma\left(L_{1}+j\right)\left(\tilde{\alpha}_{f, i}\right)^{L_{1}+j} \tilde{P}_{I}^{j}}{\Gamma(j) \Gamma\left(L_{1}+1\right)} .
\end{aligned}
$$

As can be clearly observed from (10), when $\tilde{P}_{I}$ is proportional to $\tilde{P}_{T}$ with constant $\mu$, the diversity order is zero, which completes the proof.

\section{Ergodic Capacity Analysis}

The ergodic capacity is defined as [11], [15]

$$
\begin{aligned}
C & =\int_{0}^{\infty} \log _{2}(1+x) f_{\gamma}(x) d x \\
& =\frac{1}{\log (2)} \int_{0}^{\infty} \frac{1-F_{\gamma}(x)}{1+x} d x .
\end{aligned}
$$

Substituting the CDF of the SIR in (9) into (11), yields (12) at the top of the next page. By employing a partial fraction expansion [33, Eq. 2.102], the first integral in (12) can be evaluated as

$$
\begin{aligned}
\Upsilon_{1}= & \sum_{k=0}^{l}\left(\begin{array}{l}
l \\
k
\end{array}\right)\left[\left(-\tilde{P}_{T} / \beta_{h} \tilde{P}_{I_{i}} \tilde{\alpha}_{f, i}\right)^{l-k}\right. \\
& \left(\tilde{P}_{T} / \beta_{h} \tilde{P}_{I_{i}} \tilde{\alpha}_{f, i}-1\right)^{-(l+j-k)} \log \left(\tilde{P}_{T} / \beta_{h} \tilde{P}_{I_{i}} \tilde{\alpha}_{f, i}\right)- \\
& \sum_{\tau=2}^{l+j-k} \frac{(-1)^{l-k}}{\tau-1}\left(\tilde{P}_{T} / \beta_{h} \tilde{P}_{I_{i}} \tilde{\alpha}_{f, i}-1\right)^{-(l+j-k-\tau+1)} \\
& \left.\left(\tilde{P}_{T} / \beta_{h} \tilde{P}_{I_{i}} \tilde{\alpha}_{f, i}\right)^{l-k-(\tau-1)}\right] .
\end{aligned}
$$

Based on $\mathcal{J}_{1}(x)$ in (8), and using some algebraic manipulations, we calculate the second integral in (12) as

$$
\begin{aligned}
\Upsilon_{2}= & e^{-\tilde{\beta}_{g} \mu} \sum_{p=0}^{l+\tilde{l}-1} \sum_{k=0}^{l}\left(\begin{array}{c}
l+\tilde{l}-1 \\
p
\end{array}\right)\left(\begin{array}{l}
l \\
k
\end{array}\right) \mu^{l+\tilde{l}-1-p} \\
& \left(\tilde{I}_{p} \beta_{h} \tilde{P}_{I_{i}} \tilde{\alpha}_{f, i}\right)^{j} \zeta .
\end{aligned}
$$

${ }^{3}$ The lack of diversity gain is also observed in conventional relay networks when the co-channel interference is significant compared to the transmit power [34]. 


$$
\begin{aligned}
F_{\gamma}(x)= & 1-\sum_{l=0}^{L_{1}-1} \sum_{i=1}^{M} \sum_{j=1}^{L_{2}} \Lambda_{i, j, l}\left(1 / \tilde{P}_{T}\right)^{-j} x^{l}\left(x+\frac{\tilde{P}_{T}}{\beta_{h} \tilde{P}_{I_{i}} \tilde{\alpha}_{f, i}}\right)^{-(l+j)} F_{X}(\mu)- \\
& \widetilde{\sum} \sum_{l=0}^{L_{1}-1} \sum_{i=1}^{M} \sum_{j=1}^{L_{2}} \Lambda_{i, j, l} \tilde{l}\left(x / \tilde{I}_{p}\right)^{-j} \mathcal{J}_{1}(x)+\widetilde{\sum} \sum_{l=0}^{L_{1}-1} \sum_{i=1}^{M} \sum_{j=1}^{L_{2}} \Lambda_{i, j, l} \tilde{\beta}_{g}\left(x / \tilde{I}_{p}\right)^{-j} \mathcal{J}_{2}(x) .
\end{aligned}
$$

$$
\begin{aligned}
C= & \frac{1}{\log (2)}[F_{X}(\mu) \sum_{l=0}^{L_{1}-1} \sum_{i=1}^{M} \sum_{j=1}^{L_{2}} \Lambda_{i, j, l}\left(1 / \tilde{P}_{T}\right)^{-j} \underbrace{\int_{0}^{\infty} \frac{x^{l}}{(1+x)}\left(x+\frac{\tilde{P}_{T}}{\beta_{h} \tilde{P}_{I_{i}} \tilde{\alpha}_{f, i}}\right)^{-(l+j)} d x}_{\Upsilon_{1}}+ \\
& \widetilde{\sum} \sum_{l=0}^{L_{1}-1} \sum_{i=1}^{M} \sum_{j=1}^{L_{2}} \Lambda_{i, j, l}(\underbrace{\int_{0}^{\infty}\left(x / \tilde{I}_{p}\right)^{-j} \frac{J_{1}(x)}{1+x} d x}_{\Upsilon_{2}}-\tilde{\beta}_{g}^{\left.\tilde{S}_{0}^{\infty}\left(x / \tilde{I}_{p}\right)^{-j} \frac{J_{2}(x)}{1+x} d x\right)} .
\end{aligned}
$$

Detailed derivations of (14) and $\zeta$ are provided in Appendix D.

Given that $\Upsilon_{2}$ and $\Upsilon_{3}$ share a similar form, we can directly evaluate $\Upsilon_{3}$ as

$$
\Upsilon_{3}=e^{-\tilde{\beta}_{g} \mu} \sum_{p=0}^{l+\tilde{l}} \sum_{k=0}^{l}\left(\begin{array}{c}
l+\tilde{l} \\
p
\end{array}\right)\left(\begin{array}{l}
l \\
k
\end{array}\right) \mu^{l+\tilde{l}-p}\left(\tilde{I}_{p} \beta_{h} \tilde{P}_{I_{i}} \tilde{\alpha}_{f, i}\right)^{j} \zeta .
$$

Finally, substituting $\Upsilon_{1}, \Upsilon_{2}$, and $\Upsilon_{3}$ into (12) results in a closed-form expression for the ergodic capacity. Note that we have applied the specialized treatises on Fox's $\mathrm{H}$ function in our analysis of the ergodic capacity. A similar application of Fox's H function can be found in [35] which derived the ASER of cooperative relaying in non-spectrum sharing non-single carrier systems.

\section{Asymptotic Ergodic Capacity Analysis}

In this subsection, we focus on the ergodic capacity in the region of high values of $\tilde{P}_{T}$ and where $\tilde{I}_{p}$ is proportional to $\tilde{P}_{T}$, i.e., $\mu=$ const. We first rewrite the CDF of $Y$ given in (6) as

$$
F_{Y}(y)=1-\tilde{F}_{Y}(y)
$$

where $\quad \tilde{F}_{Y}(y)=\sum_{l=0}^{L_{1}-1} \sum_{i=1}^{M} \sum_{j=1}^{L_{2}} \Lambda_{i, j, l} y^{l}(y+$ $\left.\frac{1}{\beta_{h} \tilde{P}_{I_{i}} \tilde{\alpha}_{f, i}}\right)^{-(l+j)} \mathrm{U}(y)$. Substituting (16) into (4), we have

$$
\begin{aligned}
F_{\gamma}(x)= & \left(1-\tilde{F}_{Y}\left(x / \tilde{P}_{T}\right)\right) F_{X}(\mu)+ \\
& \int_{\mu}^{\infty}\left(1-\tilde{F}_{Y}\left(x t / \tilde{I}_{p}\right)\right) f_{X}(t) d t \\
= & \left(1-\tilde{F}_{Y}\left(x / \tilde{P}_{T}\right)\right) F_{X}(\mu)+\left(1-F_{X}(\mu)\right)- \\
& \int_{\mu}^{\infty} \tilde{F}_{Y}\left(x t / \tilde{I}_{p}\right) f_{X}(t) d t \\
= & 1-\tilde{F}_{Y}\left(x / \tilde{P}_{T}\right) F_{X}(\mu)-\int_{\mu}^{\infty} \tilde{F}_{Y}\left(x t / \tilde{I}_{p}\right) f_{X}(t) d t .
\end{aligned}
$$

Now according to (11), the ergodic capacity is given by

$$
\begin{aligned}
C= & \frac{1}{\log (2)} \int_{0}^{\infty} \frac{1-F_{\gamma}(x)}{1+x} d x \\
= & \frac{1}{\log (2)}\left[F_{X}(\mu) \int_{0}^{\infty} \frac{\tilde{F}_{Y}(x)}{1 / \tilde{P}_{T}+x} d x+\right. \\
& \left.\int_{0}^{\infty} \frac{1}{1 / \tilde{I}_{p}+x} \int_{\mu}^{\infty} \tilde{F}_{Y}(x t) f_{X}(t) d t d x\right]
\end{aligned}
$$

Theorem 2: The asymptotic ergodic capacity, denoted by $C^{\infty}$, is given by

$$
\begin{aligned}
C^{\infty \triangleq} \triangleq \underset{\substack{\tilde{P}_{T} \rightarrow \infty, \tilde{I}_{p \rightarrow \infty}, \mu=\text { const }}}{C}= & \log _{2}\left(\tilde{P}_{T}\right)+\left(1-F_{X}(\mu)\right) \log _{2}(\mu)- \\
& \frac{1}{\log (2)}\left[\Theta_{1}+\widetilde{\sum} e^{-\tilde{\beta}_{g} \mu}\left(\tilde{l} \Lambda_{1}-\tilde{\beta}_{g} \Lambda_{2}\right)\right]
\end{aligned}
$$

where

$$
\begin{aligned}
\Theta_{1} \triangleq & \sum_{l=1}^{L_{1}-1} \sum_{i=1}^{M} \sum_{j=1}^{L_{2}} \frac{(-1)^{j} \theta_{i, j} l \Gamma(l)\left(\tilde{P}_{I_{i}} \tilde{\alpha}_{f, i}\right)^{j}}{\Gamma(l+1)}\left[\psi(l)-\log \left(\beta_{h}\right)-\right. \\
& \left.\psi(j)-\log \left(\tilde{P}_{I_{i}} \tilde{\alpha}_{f, i}\right)\right]-\sum_{l=0}^{L_{1}-1} \sum_{i=1}^{M} \sum_{j=1}^{L_{2}}(-1)^{j} \theta_{i, j}\left(\tilde{P}_{I_{i}} \tilde{\alpha}_{f, i}\right)^{j} \\
& {\left[\psi(l+1)-\log \left(\beta_{h}\right)-\psi(j)-\log \left(\tilde{P}_{I_{i}} \tilde{\alpha}_{f, i}\right)\right], } \\
\Lambda_{1} \triangleq & \sum_{i=0} \tilde{l}-1 \\
& \sum_{j=0}^{i} \frac{\Gamma(i+1)}{\Gamma(i-j+1)}\left((-1)^{i-j-1}\left(\mu \tilde{\beta}_{g}\right)^{i-j} e^{\mu \tilde{\beta}_{g}} \operatorname{Ei}\left(-\mu \tilde{\beta}_{g}\right)+\right. \\
& \sum_{i-j} \tilde{\beta}^{i+1}[\Gamma(i+1) \log (\mu)+ \\
& \left.\left.\sum_{k=1}^{i} \Gamma(k)\left(-\mu \tilde{\beta}_{g}\right)^{i-j-k}\right)\right], \text { and }
\end{aligned}
$$




$$
\begin{aligned}
\Lambda_{2} \triangleq & \sum_{i=0}^{\tilde{l}}\left(\begin{array}{l}
\tilde{l} \\
i
\end{array}\right) \frac{\mu^{\tilde{l}-i}}{\left(\tilde{\beta}_{g}\right)^{i+1}}\left[\Gamma(i+1) \log (\mu)+\sum_{j=0}^{i} \frac{\Gamma(i+1)}{\Gamma(i-j+1)}\right. \\
& \left((-1)^{i-j-1}\left(\mu \tilde{\beta}_{g}\right)^{i-j} e^{\mu \tilde{\beta}_{g}} \operatorname{Ei}\left(-\mu \tilde{\beta}_{g}\right)+\right. \\
& \left.\left.\sum_{k=1}^{i-j} \Gamma(k)\left(-\mu \tilde{\beta}_{g}\right)^{i-j-k}\right)\right]
\end{aligned}
$$

where $\psi(N)=-C_{u}+\sum_{n=1}^{N-1} \frac{1}{n}$ with Euler's constant $C_{u}[33$, Eq. 8.365], and $\operatorname{Ei}(\cdot)$ is the exponential integral function [33, Eq. 8.211.1].

Proof: A detailed derivation of (19) is provided in Appendix E.

With the help of (19), we confirm that the multiplexing gain [36] is 1 in bits/sec/Hz/(3 dB), which is given by

$$
\mathcal{S}^{\infty}=\lim _{\tilde{P}_{T} \rightarrow \infty} \frac{C^{\infty}}{\log _{2} \tilde{P}_{T}}=1 .
$$

Moreover, using (19), we can easily calculate the additional power cost for different network parameters while maintaining a specified target ergodic capacity. For example, in light of the interference power from the PU-Txs on the SU-Rx, we consider two interference power scenarios: 1) $\tilde{P}_{I_{1}}=\cdots=\tilde{P}_{I_{M}}=\tilde{P}_{I}^{1}$, and 2) $\tilde{P}_{I_{1}}=\cdots=\tilde{P}_{I_{M}}=\tilde{P}_{I}^{2}$ with $\tilde{P}_{I}^{1}<$ $\tilde{P}_{I}^{2}$. Compared to the former, the additional power cost for achieving the specified target ergodic capacity in the second scenario is calculated as

$$
\Delta \tilde{P}_{T}(\mathrm{~dB})=\frac{10}{\log (10)}\left[\Theta_{1}\left(\tilde{P}_{I}^{2}\right)-\Theta_{1}\left(\tilde{P}_{I}^{1}\right)\right] .
$$

\section{Simulation Results}

In this section, we validate our analytical results and consider the impact of multiple primary transceivers on the outage probability and ergodic capacity of cognitive underlay single carrier systems under different interference power profiles. We set $Q=256$ and $Q_{g}=16$ as the data symbol block size and the CP length, respectively. We use BPSK modulation and a fixed $\gamma_{t h}=1 \mathrm{~dB}$ in the computation of the outage probability. Recall that $\tilde{I}_{p}=\frac{I_{p}}{\sigma_{n}^{2}}$ and $\tilde{P}_{T}=\frac{P_{T}}{\sigma_{n}^{2}}$. We assume that the SU-Tx and SU-Rx are placed at $[0,0]$ and $[1,0]$, respectively. We consider $M=4$ PU-Txs which are placed at $[0.3,0.3]$, $[0.4,0.3],[0.5,0.3]$, and $[0.6,0.3]$, respectively. Similarly, we consider $N=2$ PU-Rxs which are placed at $[0.4,0.5]$ and $[0.6,0.5]$, respectively. The pathloss component for the channel between any two nodes $\mathrm{B}$ and $\mathrm{C}$ is exponentially decaying as $\alpha^{\mathrm{B}, \mathrm{C}}=d_{\mathrm{B}, \mathrm{C}}^{-\epsilon}$, where $d_{\mathrm{B}, \mathrm{C}}$ is the distance between $\mathrm{B}$ and $\mathrm{C}$, and $\epsilon$ is the path loss exponent. In all simulation scenarios, we assume $\epsilon=4$ with the same interference power from all the PU-Txs such that $\tilde{P}_{I}=1$. In the following simulation figures, the curves obtained from actual link simulations are denoted by $\mathbf{E x}$, analytically derived curves are denoted by $\mathbf{A n}$, and asymptotically obtained curves are denoted by As.

\section{A. Outage Probability: Proportional Interference Power Con- straint}

Figs. 2 and 3 show the outage diversity gain with proportional interference power constraint. To improve the clarity of

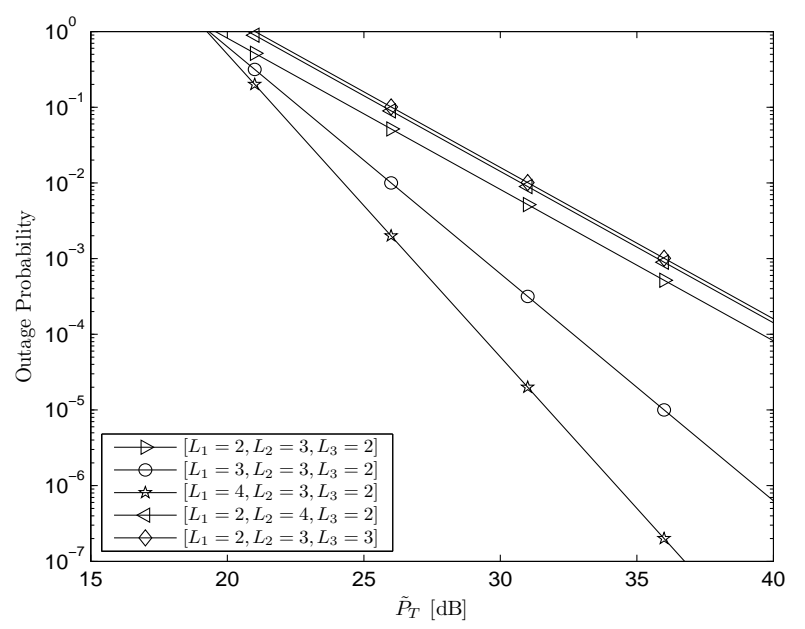

Fig. 2. Outage probability for various values of $\left(L_{1}, L_{2}, L_{3}\right)$ with $(M=$ $3, N=2)$.

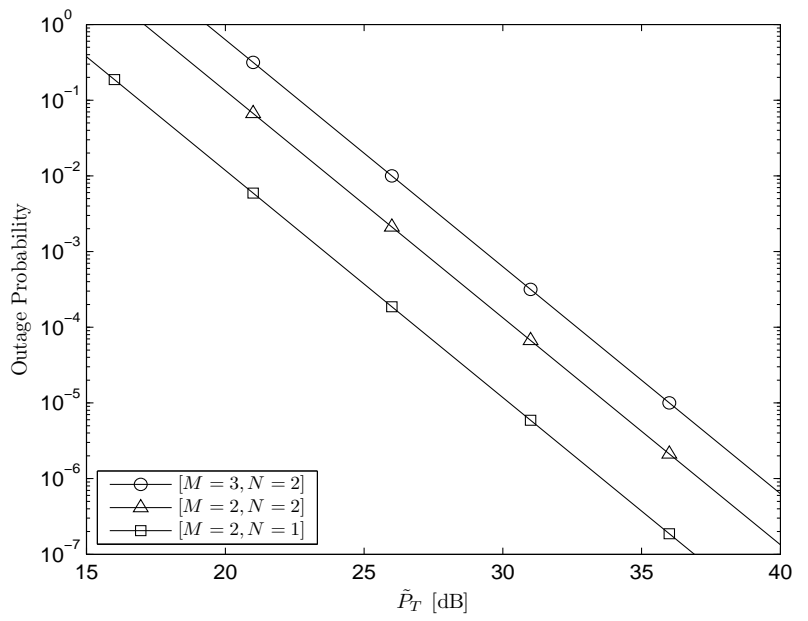

Fig. 3. Outage probability for various values of $(M, N)$ with $\left(L_{1}=3, L_{2}=\right.$ $\left.3, L_{3}=2\right)$.

the plots, we will only show asymptotic outage curves. From Figs. 2 and 3, we observe the following.

1) For a fixed $(M=3, N=2)$, three different frequency selective fading scenarios of $\left(L_{1}=2, L_{2}=3, L_{3}=\right.$ $2),\left(L_{1}=2, L_{2}=4, L_{3}=2\right)$, and $\left(L_{1}=2, L_{2}=\right.$ $\left.3, L_{3}=3\right)$ display the same outage diversity gain in the large $\tilde{P}_{T}$ regime. In addition, for a fixed $(M=3, N=$ 2, $L_{2}=3, L_{3}=2$ ), the multipath gain of $L_{1}=4$ has the best outage probability compared with $L_{1}=2$ and $L_{1}=3$. It can be seen that their slopes are proportional to the value of $L_{1}$. The difference between their outage diversity gains is readily apparent as $\tilde{P}_{T}$ increases.

2) From Fig. 3, we can see that three different primary transceiver combinations of $(M=3, N=2),(M=$ $2, N=2)$, and $(M=1, N=1)$ for a fixed $\left(L_{1}=3, L_{2}=3, L_{3}=2\right)$ display the same outage diversity gain in the large $\tilde{P}_{T}$ regime. Note that the 


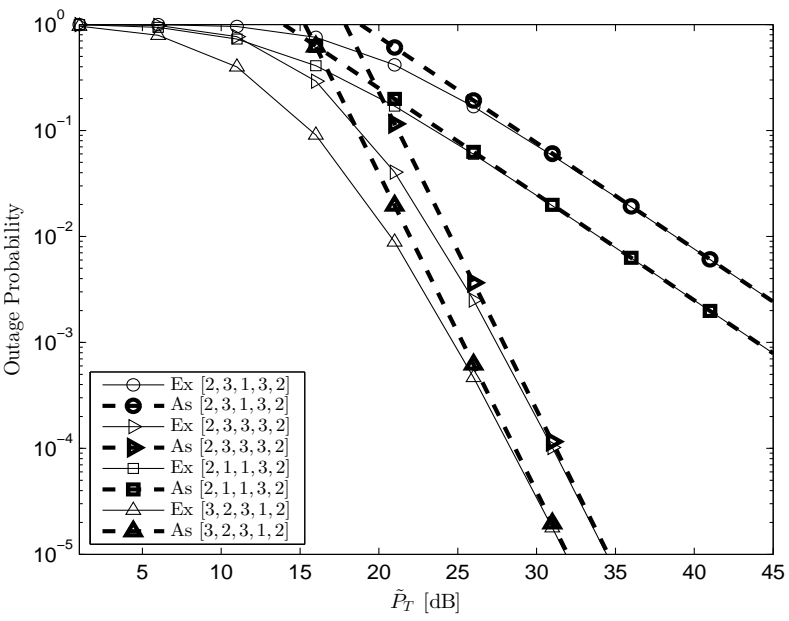

Fig. 4. Outage probability for various values of $\left(M, N, L_{1}, L_{2}, L_{3}\right)$.

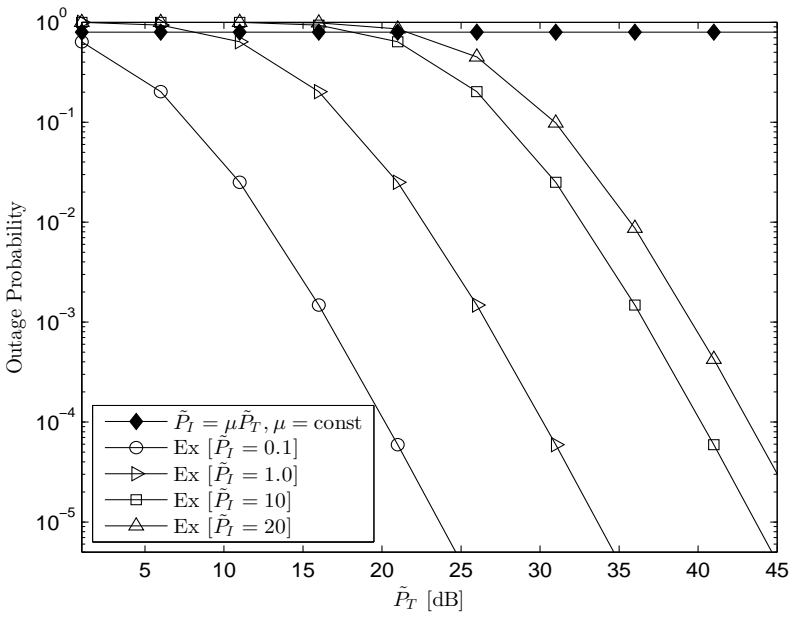

Fig. 5. Outage probability for various values of $\tilde{P}_{I}$ at fixed value of $(M=$ $\left.2, N=2, L_{1}=3, L_{2}=3, L_{3}=2\right)$.

outage probability decreases with decreasing $M$ and $N$.

From these observations, we can verify that the outage diversity gain is proportional to the multipath channel length of the frequency selective fading channel between the SUTx and SU-Rx. Other key parameters such as the number of PU-Txs and PU-Rxs, and the multipath channel lengths of the frequency selective fading channels between the SUs and PUs have no impact on the diversity gain and only impact the coding gain.

Fig. 4 compares the asymptotic outage diversity gain with the exact outage probability. As $\tilde{P}_{T} \rightarrow \infty$, the difference between the exact outage probability and the asymptotic outage probability becomes negligible for a general value of $\left(M, N, L_{1}, L_{2}, L_{3}\right)$. As in Fig. 2, the asymptotic outage diversity gain $G_{d}=L_{1}$ is clearly seen from the simulations.

Fig. 5 shows the exact and asymptotic outage probability for various values of $\tilde{P}_{I}$ at a fixed $\left(M=2, N=2, L_{1}=3, L_{2}=\right.$ $\left.3, L_{3}=2\right)$. We see that as $\tilde{P}_{I}$ increases, a lower outage

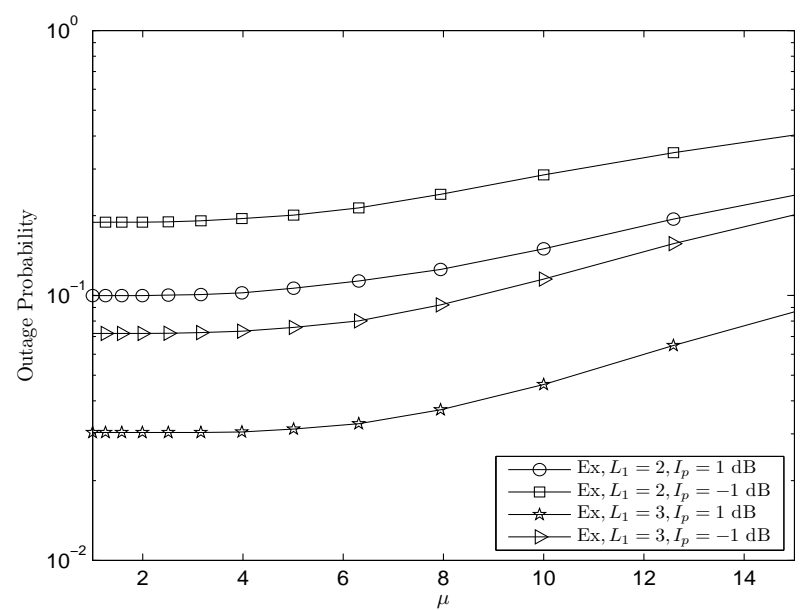

Fig. 6. Outage probability for various values of $L_{1}$ at fixed value of $I_{p}$ and $\left(M=2, N=1, L_{2}=2, L_{3}=3\right)$.

probability is obtained. This figure shows that when $\mu=$ const and $\tilde{P}_{I}$ is proportional to $\tilde{P}_{T}$, the diversity gain is zero. However, when $\tilde{P}_{I}$ is not proportional to $\tilde{P}_{T}$, the diversity gain of $G_{d}=L_{1}$ is achieved, as predicted in Corollary 1 .

\section{B. Outage Probability: Fixed Interference Power Constraint}

In Fig. 6, we plot the outage probability with fixed interference power constraint. Since $\gamma_{\mathrm{SINR}}=$ $\min \left(\tilde{P}_{T}, \frac{\tilde{I}_{p}}{\max _{k=1, \ldots, N}\left\{\tilde{\alpha}_{g, k}\left\|\boldsymbol{g}_{k}\right\|^{2}\right\}}\right) \approx \frac{\tilde{I}_{p}}{\max _{k=1, \ldots, N}\left\{\tilde{\alpha}_{g, k}\left\|\boldsymbol{g}_{k}\right\|^{2}\right\}}$ as $\mu$ decreases, $\tilde{I}_{p}=I_{p} / \sigma_{n}^{2}$ dominates the $\gamma_{\mathrm{SINR}}$. Thus, for $\left(M=2, N=1, L_{2}=2, L_{3}=3\right)$ and $L_{1}=\{2,3\}$, we see that an outage probability floor occurs when $\mu<3$ and $\tilde{I}_{p}$ is fixed. Although the outage probability decreases with increasing $\tilde{I}_{p}$ or $L_{1}$, an outage probability floor is unavoidable as in [12] and [14]. Confirming our result in (10), we find that the asymptotic outage diversity gain is zero when the interference power constraint is fixed.

\section{Ergodic Capacity}

In Fig. 7, we show the exact and analytical ergodic capacity for various values of $L_{1}$ at fixed $(M=2, N=$ $\left.2, L_{2}=3, L_{3}=3\right)$. We consider two cases of $\left.\tilde{P}_{T}: 1\right) \tilde{P}_{T}$ proportional to $\tilde{I}_{p}$, which is specified by $\mu=$ const; and 2) $\tilde{P}_{T}$ is fixed and independent of $\tilde{I}_{p}$, which is specified by $\mu=$ varying. This figure shows that when $\mu$ is constant, the ergodic capacity increases with increasing $L_{1}$ due to a higher multipath gain. However, for a fixed value of $\tilde{P}_{T_{\tau}}=10 \mathrm{~dB}$, as $\tilde{I}_{p}$ increases, $\tilde{P}_{T}$ dominates $\min \left(\tilde{P}_{T}, \frac{\tilde{I}_{p}}{\max _{k=1, \ldots, N}\left\{\tilde{\alpha}_{g, k}\left\|\boldsymbol{g}_{k}\right\|^{2}\right\}}\right)$, since $\min \left(\tilde{P}_{T}, \frac{\tilde{I}_{p}}{\max _{k=1, \ldots, N}\left\{\tilde{\alpha}_{g, k}\left\|\boldsymbol{g}_{k}\right\|^{2}\right\}}\right) \approx \tilde{P}_{T}$. Thus, we observe that although a higher ergodic capacity is achieved depending on the size of $L_{1}$, it enters a saturation region for a fixed value of $\tilde{P}_{T}$. 


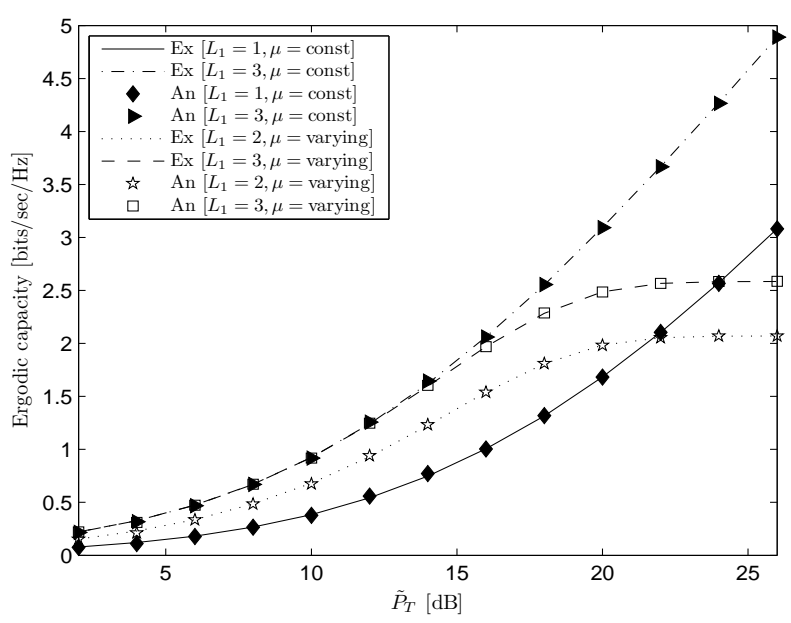

Fig. 7. Ergodic capacity for various values of $L_{1}$ and $\tilde{I}_{p}$ for $(M=2, N=$ $\left.2, L_{2}=3, L_{3}=3\right)$.

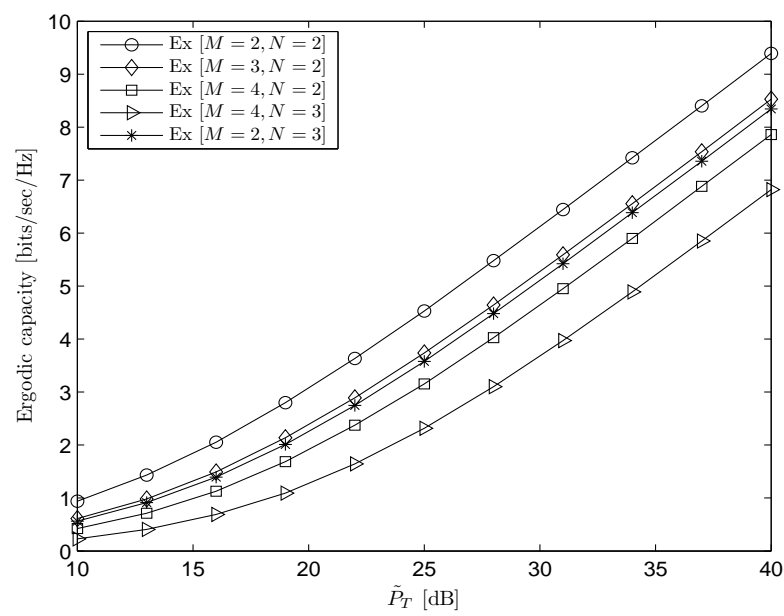

Fig. 8. Ergodic capacity for various values of $M$ and $N$ for $\left(L_{1}=2, L_{2}=\right.$ $\left.2, L_{3}=2\right)$.

In Fig. 8, we investigate the effect of the number of PU-Txs and PU-Rxs. In this figure, we plot the exact ergodic capacity for a constant $\mu$ and $\left(L_{1}=2, L_{2}=2, L_{3}=2\right)$. We can readily see that as the number of PU-Txs increases, the ergodic capacity degrades due to increasing interference. For example, $(M=2, N=2)$ vs. $(M=3, N=2)$. In addition, as the number of PU-Rxs increases, a degraded ergodic capacity is achieved due to greater restriction on peak interference power at the SU-Tx. For example, note $(M=2, N=2)$ vs. $(M=$ $2, N=3)$ and $(M=4, N=2)$ vs. $(M=4, N=3)$.

In Fig. 9, we plot the ergodic capacity versus $\tilde{P}_{I}$ for fixed values of $\left(M=4, N=2, L_{1}=2, L_{2}=2, L_{3}=2\right)$. As expected, the ergodic capacity deteriorates with increasing $\tilde{P}_{I}$. All the curves are parallel, which is implied by (21). As $\tilde{P}_{T}$ increases, the differences between the exact ergodic capacities and the asymptotic ergodic capacities become negligible. Given a specified target ergodic capacity, the additional power

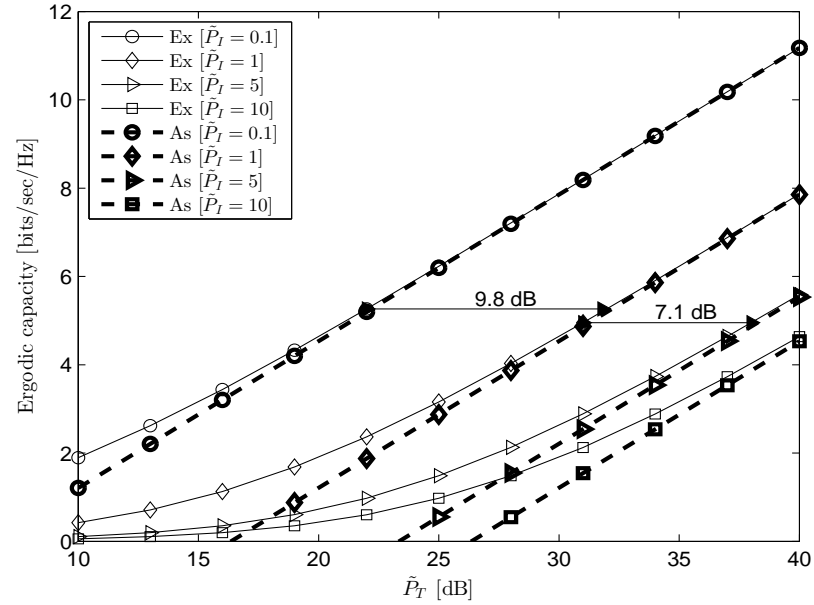

Fig. 9. Ergodic capacity for various values of $\tilde{P}_{I}$ for $\left(M=4, N=2, L_{1}=\right.$ $2, L_{2}=2, L_{3}=2$ ).

cost $\Delta \tilde{P}_{T}$ for different $\tilde{P}_{I}$ is accurately calculated using (22). At $5.26 \mathrm{bits} / \mathrm{sec} / \mathrm{Hz}, \tilde{P}_{I}=1$ requires an additional $9.8 \mathrm{~dB}$ power cost over $\tilde{P}_{I}=0.1$. Also, at $4.95 \mathrm{bits} / \mathrm{sec} / \mathrm{Hz}$, an additional $7.1 \mathrm{~dB}$ power cost is required for $\tilde{P}_{I}=5$ over $\tilde{P}_{I}=1$.

\section{CONCLUSiOns}

In this paper, the joint impact of multiple PU-Txs and PURxs has been examined for cognitive single carrier systems with underlay spectrum-sharing. We have presented a general framework for cognitive networks over frequency selective fading channels with multiple primary transceivers by statistically characterizing the end-to-end SINR and SIR at the SU-Rx. Then, we have derived new closed-form analytical expressions for the outage probability and ergodic capacity. The asymptotic outage probability and asymptotic ergodic capacity have also been proposed to provide further insights into the impact of the power constraints and system parameters on the secondary system performance. Several important phenomena have been highlighted. Specifically, we have shown that irrespective of the interference from the PU-Txs, cognitive single carrier systems display an asymptotic diversity gain which is equal to the channel impulse length of the secondary network. However, when the interference power from the PUTxs is proportional to the maximum transmit power at the SUTx, we establish that the asymptotic diversity gain diminishes to zero. Based on asymptotic ergodic capacity, we have shown that the multiplexing gain is constant and have accurately calculated the power cost for different network parameters while maintaining a specified target ergodic capacity. 


\section{APPENDIX A: DERIVATION OF LEMMA 1}

The PDF and the CDF of the gamma distributed RV $A$ are, respectively, given by

$$
\begin{aligned}
f_{A}(x) & =\frac{\beta_{h}^{L_{1}}}{\Gamma\left(L_{1}\right)} x^{L_{1}-1} e^{-\beta_{h} x} \mathrm{U}(x) \text { and } \\
F_{A}(x) & =\left(1-e^{-\beta_{h} x} \sum_{i=0}^{L_{1}-1} \frac{\left(\beta_{h} x\right)^{i}}{i !}\right) \mathrm{U}(x) .
\end{aligned}
$$

According to the derivations provided in [37], the PDF of the $\mathrm{RV} B \triangleq \sum_{k=1}^{M} \tilde{P}_{I_{k}} \tilde{\alpha}_{f, k}\left\|\boldsymbol{f}_{k}\right\|^{2}$ is given by

$$
f_{B}(x)=\sum_{i=1}^{M} \sum_{j=1}^{L_{2}} \frac{(-1)^{j} \theta_{i, j}}{\Gamma(j)} x^{j-1} e^{-\frac{x}{\tilde{P}_{I_{i}} \tilde{\alpha}_{f, i}}} \mathrm{U}(x) .
$$

Now using Eqs. (A.1) and (A.2), the CDF of the RV $Y$ is

$$
\begin{aligned}
F_{Y}(y)= & \int_{0}^{\infty} f_{B}(x) F_{A}(y x) d x \\
= & 1-\sum_{l=0}^{L_{1}-1} \frac{\left(\beta_{h}\right)^{l} y^{l}}{\Gamma(l+1)} \int_{0}^{\infty} f_{B}(x) e^{-\beta_{h} y x}(x)^{l} d x \\
= & 1-\sum_{l=0}^{L_{1}-1} \frac{\left(\beta_{h}\right)^{l} y^{l}}{\Gamma(l+1)} \sum_{i=1}^{M} \sum_{j=1}^{L_{2}} \frac{(-1)^{j} \theta_{i, j}}{\Gamma(j)} \\
& \int_{0}^{\infty} e^{-x}\left(\beta_{h} y+\frac{1}{\tilde{P}_{I_{i}} \tilde{\alpha}_{f, i}}\right) \\
= & 1-\sum_{l=0}^{L_{1}-1} \sum_{i=1}^{M} \sum_{j=1}^{L_{2}} \frac{(-1)^{j}\left(\beta_{h}\right)^{-j} \theta_{i, j} \Gamma(l+j)}{\Gamma(l+1) \Gamma(j)} \\
& y^{l}\left(y+\frac{1}{\beta_{h} \tilde{P}_{I_{i}} \tilde{\alpha}_{f, i}}\right)^{-(l+j)} \mathrm{U}(y)
\end{aligned}
$$

which proves (6).

\section{APpendix B: DeRivation of (7)}

For the final form of (4), we need to compute

$$
\mathcal{I}_{1}(x)=\int_{\mu}^{\infty} F_{Y}\left(x t / \tilde{I}_{p}\right) f_{X}(t) d t
$$

which is evaluated as

$$
\begin{aligned}
I_{1}(x)= & 1-F_{X}(\mu)-\widetilde{\sum} \sum_{l=0}^{L_{1}-1} \sum_{i=1}^{M} \sum_{j=1}^{L_{2}} \Lambda_{i, j, l}\left[\tilde{l}\left(x / \tilde{I}_{p}\right)^{-j}\right. \\
& \underbrace{\left.\int_{\mu}^{\infty} t^{l+\tilde{l}-1} e^{-\tilde{\beta}_{g} t}\left(t+\frac{\tilde{I}_{p}}{\beta_{h} \tilde{P}_{I_{i}} \tilde{\alpha}_{f, i} x}\right)^{-(l+j)} d t\right]}_{\mathcal{J}_{1}(x)}+ \\
& \underbrace{\sum_{\mu} \sum_{l=0}^{L_{1}-1} \sum_{i=1}^{M} \sum_{j=1}^{L_{2}} \Lambda_{i, j, l}\left[\tilde{\beta}_{g}\left(x / \tilde{I}_{p}\right)^{-j}\right.}_{\mathcal{J}_{2}(x)} e^{-\tilde{\beta}_{g} t}\left(t+\frac{\tilde{I}_{p}}{\beta_{h} \tilde{P}_{I_{i}} \tilde{\alpha}_{f, i} x}\right)^{-(l+j)} d t]
\end{aligned}
$$

With some computations, we can obtain $\mathcal{J}_{1}(x)$ and $\mathcal{J}_{2}(x)$.

\section{APPENDIX C: A PROOF OF THEOREM 1}

We start the derivation by rewriting the definition of $F_{Y}\left(x / \tilde{P}_{T}\right)$, which is given by

$$
\begin{aligned}
F_{Y}\left(x / \tilde{P}_{T}\right) & =\operatorname{Pr}\left(A<x B / \tilde{P}_{T}\right) \\
& =E_{B}\left\{F_{A}\left(x B / \tilde{P}_{T} \mid B\right)\right\} .
\end{aligned}
$$

For a frequency selective fading channel, $F_{A}\left(x B / \tilde{P}_{T} \mid B\right)$ can be approximated as $F_{A}\left(\frac{x B}{\tilde{P}_{T}} \mid B\right)^{\tilde{P}_{T} \rightarrow \infty} \frac{1}{\Gamma\left(L_{1}+1\right)}\left(\frac{\beta_{h} x B}{\tilde{P}_{T}}\right)^{L_{1}}$ [12]; thus an asymptotic $F_{Y}\left(x / \tilde{P}_{T}\right)$ is given by

$$
\begin{aligned}
& \tilde{F}_{Y}\left(x / \tilde{P}_{T}\right)= \\
& \sum_{i=1}^{M} \sum_{j=1}^{L_{2}} \frac{(-1)^{j} \theta_{i, j}\left(\beta_{h}\right)^{L_{1}} \Gamma\left(L_{1}+j\right)\left(\tilde{P}_{I_{i}} \tilde{\alpha}_{f, i}\right)^{L_{1}+j}}{\Gamma(j) \Gamma\left(L_{1}+1\right)}\left(\frac{x}{\tilde{P}_{T}}\right)^{L_{1}} \\
& =\Omega_{1}\left(\frac{x}{\tilde{P}_{T}}\right)^{L_{1}} .
\end{aligned}
$$

Using (C.2), an asymptotic $\mathcal{I}_{1}(x)$ can be evaluated as

$$
\begin{aligned}
\tilde{\mathcal{I}}_{1}(x) & =\int_{\mu}^{\infty} \tilde{F}_{Y}\left(x t / \tilde{I}_{p}\right) f_{X}(t) d t \\
& =\Omega_{1}\left(x / \mu \tilde{P}_{T}\right)^{L_{1}} \int_{\mu}^{\infty} t^{L_{1}} f_{X}(t) d t .
\end{aligned}
$$

Replacing $f_{X}(t)$ with the corresponding expression in (5), we have

$$
\begin{aligned}
\tilde{\mathcal{I}}_{1}(x)= & \int_{\mu}^{\infty} \tilde{F}_{Y}\left(x t / \tilde{I}_{p}\right) f_{X}(t) d t \\
= & \Omega_{1}\left(\frac{1}{\mu}\right)^{L_{1}} \widetilde{\sum}\left[\tilde{l}\left(\tilde{\beta}_{g}\right)^{-\left(L_{1}+\tilde{l}\right)} \Gamma\left(L_{1}+\tilde{l}, \mu \tilde{\beta}_{g}\right)-\right. \\
& \left.\tilde{\beta}_{g}\left(\tilde{\beta}_{g}\right)^{-\left(L_{1}+\tilde{l}+1\right)} \Gamma\left(L_{1}+\tilde{l}+1, \mu \tilde{\beta}_{g}\right)\right]\left(\frac{x}{\tilde{P}_{T}}\right)^{L_{1}} .
\end{aligned}
$$

Now using (C.2) and (C.4), we can readily obtain the following:

$$
\begin{gathered}
P_{\text {out }}\left(\gamma_{\text {th }}\right) \underset{\substack{\tilde{P}_{T} \rightarrow \infty \\
\tilde{I}_{p} \rightarrow \infty, \mu=\text { const }}}{\approx} \Omega_{1}\left(F_{X}(\mu)+(1 / \mu)^{L_{1}} \widetilde{\sum}\left[\tilde{l}\left(\tilde{\beta}_{g}\right)^{-\left(L_{1}+\tilde{l}\right)}\right.\right. \\
\Gamma\left(L_{1}+\tilde{l}, \mu \tilde{\beta}_{g}\right)-\left(\tilde{\beta}_{g}\right)^{-\left(L_{1}+\tilde{l}\right)} \\
\left.\left.\Gamma\left(L_{1}+\tilde{l}+1, \mu \tilde{\beta}_{g}\right)\right]\right)\left(\gamma_{\mathrm{th}} / \tilde{P}_{T}\right)^{L_{1}} \quad(\mathrm{C} .5) \\
\text { where } \Omega_{1} \triangleq \sum_{i=1}^{M} \sum_{j=1}^{L_{2}} \frac{(-1)^{j} \theta_{i, j}\left(\beta_{h}\right)^{L_{1}} \Gamma\left(L_{1}+j\right)\left(\tilde{P}_{I_{i}} \tilde{\alpha}_{f, i}\right)^{L_{1}+j}}{\Gamma(j) \Gamma\left(L_{1}+1\right)}
\end{gathered}
$$

and $\Gamma(\cdot, \cdot)$ denotes the incomplete gamma function. Thus, it can be seen that the outage diversity gain as a function of $\left(\gamma_{\mathrm{th}} / \tilde{P}_{T}\right)$ is given by $G_{d}=L_{1}$. Similarly, the outage probability at a fixed $\tilde{P}_{T}$ is given by

$$
\begin{aligned}
P_{\text {out }}\left(\gamma_{\mathrm{th}}\right) \approx & \Omega_{2} \mu^{L_{1}}+\Omega_{2} \widetilde{\sum}\left[\tilde{l}\left(\tilde{\beta}_{g}\right)^{-\left(L_{1}+\tilde{l}\right)} \Gamma\left(L_{1}+\tilde{l}, \mu \tilde{\beta}_{g}\right)-\right. \\
& \left.\left(\tilde{\beta}_{g}\right)^{-\left(L_{1}+\tilde{l}\right)} \Gamma\left(L_{1}+\tilde{l}+1, \mu \tilde{\beta}_{g}\right)\right]
\end{aligned}
$$

where $\Omega_{2} \triangleq \Omega_{1}\left(\gamma_{\mathrm{th}} / \tilde{I}_{p}\right)$. From the definition of the outage diversity gain, (C.6) shows no outage diversity gain. 


\section{APPENDIX D: DERIVATION OF (14)}

According to (8) and (12), we rewrite $\Upsilon_{2}$ as

$$
\Upsilon_{2}=e^{-\tilde{\beta}_{g} \mu} \sum_{p=0}^{l+\tilde{l}-1}\left(\begin{array}{c}
l+\tilde{l}-1 \\
p
\end{array}\right) \mu^{l+\tilde{l}-1-p}\left(\tilde{I}_{p}\right)^{j} \hbar
$$

where

$$
\begin{aligned}
\hbar \triangleq & \int_{0}^{\infty} \frac{x^{-j}}{1+x}\left(\mu+\frac{\tilde{I}_{p}}{\beta_{h} \tilde{P}_{I_{i}} \tilde{\alpha}_{f, i} x}\right)^{p-j-l+1} \\
& \int_{0}^{\infty} t^{p} e^{-\tilde{\beta}_{g}\left(\mu+\frac{\tilde{I}_{p}}{\beta_{h} \tilde{P}_{I_{i}} \tilde{\alpha}_{f, i} x}\right) t}(1+t)^{-(j+l)} d t d x .
\end{aligned}
$$

Changing variables and the order of integration, $\hbar$ is transformed as

$$
\begin{aligned}
\hbar= & \int_{0}^{\infty} t^{p} e^{-\tilde{\beta}_{g} t}(\mu+t)^{-(j+l)} \\
& \int_{0}^{\infty} \frac{x^{l}}{1+x}\left(x+\frac{\tilde{I}_{p}}{\beta_{h} \tilde{P}_{I_{i}} \tilde{\alpha}_{f, i}(\mu+t)}\right)^{-(j+l)} d x d t .
\end{aligned}
$$

From $\Upsilon_{2}$ in (12) and (13), the interior integral can be easily evaluated by substituting $\frac{\tilde{I}_{p}}{\beta_{h} \tilde{P}_{I_{i}} \tilde{\alpha}_{f, i}(\mu+t)}$ for $\frac{\tilde{P}_{T}}{\beta_{h} \tilde{P}_{I_{i}} \alpha_{f, i}}$. Hence, we derive (D.3) as (D.4) at the top of the following page. To evaluate integral $\Xi_{1}$ in (D.4), we use the following identity [38, pp. 152]:

$$
\left(1+d_{i} x\right)^{-v_{i}}=\frac{1}{\Gamma\left(v_{i}\right)} \mathrm{H}_{1,1}^{1,1}\left[\left.d_{i} x\right|_{(0,1)} ^{\left(1-v_{i}, 1\right)}\right]
$$

where $\mathrm{H}_{A, B}^{M, N}[\cdot]$ denotes Fox's H-function [39, Eq. 8.3.1.1]. Using (D.5) and [38, Eq. 2.6.2], we obtain $\Xi_{1}$ as

$$
\begin{aligned}
\Xi_{1}= & \frac{\mu^{-l}\left(\tilde{\beta}_{g}\right)^{-(p+1)}}{\Gamma(l) \Gamma(l+j-k)} \mathrm{H}_{1,(1: 1), 0,(1: 1)}^{1,1,1,1,1} \\
& {\left[\begin{array}{c|c}
\left(\mu \tilde{\beta}_{g}\right)^{-1} & (1+p, 1) \\
\frac{\beta_{h} \tilde{P}_{I_{i}} \tilde{\alpha}_{f, i}}{\left(\beta_{h} \tilde{P}_{I_{i}} \tilde{\alpha}_{f, i \mu}-\tilde{I}_{p}\right) \tilde{\beta}_{g}} & (1-l) ;(1-l-j+k, 1) \\
(0,1) ;(0,1)
\end{array}\right] . }
\end{aligned}
$$

By employing [38, Eq. 2.3.2], (D.6) can be simplified in terms of the Kampé de Feriét's function, which is reflected in (D.9) at the top of the page after next. Using a partial fraction expansion [33, Eq. (2.102)], the identity in (D.5), $\log (1+z)=\mathrm{H}_{2,2}^{1,0}\left[z \mid \begin{array}{l}(1,1),(1,1) \\ (1,1),(0,1)\end{array}\right]$ [38, pp. 152], and [38, Eq. 2.6.2], $\Xi_{2}$ in (D.4) is obtained as (D.7) which is provided on the next page. According to (D.4) and (D.6), we can directly obtain $\Xi_{3}$ in (D.4) as (D.8) on the next page. Substituting (D.6), (D.7), and (D.8) into (D.4), we obtain $\hbar$. Substituting $\hbar$ into (D.1), we arrive at $\zeta$, which is expressed in (D.9). In (D.9), $\mathrm{F}_{\rho: \sigma}^{\varpi: \vartheta}[\cdot]$ is the Kampé de Feriét function [35] and $\mathrm{H}_{E,(A: C), F,(B: D)}^{K, N, N^{\prime}, M, M^{\prime}}[\cdot]$ is the generalized Fox's Hfunction [38, Eq. (2.2.1)]. We also define $\Delta_{2,1} \triangleq 2 l+j-k-v_{1}$, $\Delta_{2,2} \triangleq 2 l+j-k-v_{2}$, and $\Delta_{1,2} \triangleq l+j-k-v_{2}$.

\section{APPENDIX E: DERIVATION OF (19)}

Using integration by parts, (19) is given by

$$
\begin{aligned}
C^{\infty}= & \frac{1}{\log (2)}\left[\log \left(\tilde{P}_{T}\right)+\left(1-F_{X}(\mu)\right) \log (\mu)-\right. \\
& F_{X}(\mu) \underbrace{\int_{0}^{\infty} \tilde{f}_{Y}(x) \log \left(1 / \tilde{P}_{T}+x\right) d x}_{\Theta_{1}}- \\
& \underbrace{\int_{0}^{\infty} \log \left(1 / \tilde{I}_{p}+x\right) \int_{\mu}^{\infty} t \tilde{f}_{Y}(x t) f_{X}(t) d t d x}_{\Theta_{2}}]
\end{aligned}
$$

where $\tilde{f}_{Y}(x)$ is obtained through the derivative of $\tilde{F}_{Y}(x)$; that is, from (A.3), we obtain $\tilde{f}_{Y}(x)$ as

$$
\begin{aligned}
\tilde{f}_{Y}(x)= & \sum_{l=1}^{L_{1}-1} \frac{\left(\beta_{h}\right)^{l} l x^{l-1}}{\Gamma(l+1)} \sum_{i=1}^{M} \sum_{j=1}^{L_{2}} \frac{(-1)^{j} \theta_{i, j}}{\Gamma(j)} \\
& \left.\int_{0}^{\infty} e^{-t\left(\beta_{h} x+\frac{1}{\tilde{P}_{I_{i}}} \tilde{\tilde{\alpha}}_{f, i}\right.}\right)(t)^{l+j-1} d t- \\
& \sum_{l=0}^{L_{1}-1} \frac{\left(\beta_{h}\right)^{l+1} x^{l}}{\Gamma(l+1)} \sum_{i=1}^{M} \sum_{j=1}^{L_{2}} \frac{(-1)^{j} \theta_{i, j}}{\Gamma(j)} \\
& \int_{0}^{\infty} e^{-t\left(\beta_{h} x+\frac{1}{\tilde{P}_{I_{i}} \tilde{\alpha}_{f, i}}\right)}(t)^{l+j} d t .
\end{aligned}
$$

As $\tilde{P}_{T} \rightarrow \infty, \Theta_{1}$ becomes asymptotically

$$
\Theta_{1}=\int_{0}^{\infty} \tilde{f}_{Y}(x) \log (x) d x .
$$

Substituting (E.2) into (E.3) and changing the order of integration, after some algebraic manipulations, we obtain (E.4) on the page after next. In (E.4), $\int_{0}^{\infty} x^{\nu-1} e^{-\mu x} \log (x) d x=\frac{1}{\mu^{\nu}} \Gamma(\nu)[\psi(\nu)-\ln \mu] \quad$ [33, Eq. 4.352.1] is employed in the derivation of $\Theta_{1}$. Likewise, $\Theta_{2}$ is given asymptotically by

$$
\Theta_{2}=\int_{0}^{\infty} \log (x) \int_{\mu}^{\infty} t \tilde{f}_{Y}(x t) f_{X}(t) d t d x .
$$

Changing the order of integration, we compute the integral in (E.5) as

$$
\begin{aligned}
\Theta_{2} & =\int_{\mu}^{\infty} t f_{X}(t) \int_{0}^{\infty} \tilde{f}_{Y}(x t) \log (x) d x d t \\
& =\int_{\mu}^{\infty} f_{X}(t)\left[\int_{0}^{\infty} \tilde{f}_{Y}(x) \log \left(\frac{x}{t}\right) d x\right] d t \\
& =\int_{\mu}^{\infty} f_{X}(t)\left[\int_{0}^{\infty} \tilde{f}_{Y}(x) \log (x) d x+\log (t)\right] d t \\
& =\int_{\mu}^{\infty} f_{X}(t)\left(\Theta_{1}+\log t\right) d t \\
& =\Theta_{1}\left(1-F_{X}(\mu)\right)+\underbrace{\int_{\mu}^{\infty} f_{X}(t) \log (t) d t}_{\Lambda} .
\end{aligned}
$$

Now we need to compute $\Lambda$ as (E.7) on the page after next. Using the binomial expansion and [33, Eq. 4.337.5], we 


$$
\begin{aligned}
\hbar= & \sum_{k=0}^{l}\left(\begin{array}{l}
l \\
k
\end{array}\right)\left(\beta_{h} \tilde{P}_{I_{i}} \tilde{\alpha}_{f, i}\right)^{j}\left[( - \tilde { I } _ { p } ) ^ { l - k } ( \tilde { I } _ { p } - \beta _ { h } \tilde { P } _ { I _ { i } } \tilde { \alpha } _ { f , i } \mu ) ^ { - ( l + j - k ) } \left(\log \left(\tilde{I}_{p} / \beta_{h} \tilde{P}_{I_{i}} \tilde{\alpha}_{f, i} \mu\right)\right.\right. \\
& \underbrace{\int_{0}^{\infty} t^{p} e^{-\tilde{\beta}_{g} t}(\mu+t)^{-l}\left(1+\frac{\beta_{h} \tilde{P}_{I_{i}} \tilde{\alpha}_{f, i}}{\beta_{h} \tilde{P}_{I_{i}} \tilde{\alpha}_{f, i} \mu-\tilde{I}_{p}} t\right)^{-(l+j-k)} d t}_{\Xi_{1}} \\
& \underbrace{\int_{0}^{\infty} t^{p} e^{-\tilde{\beta}_{g} t}(\mu+t)^{-l}\left(1+\frac{\beta_{h} \tilde{P}_{I_{i}} \tilde{\alpha}_{f, i}}{\beta_{h} \tilde{P}_{I_{i}} \tilde{\alpha}_{f, i} \mu-\tilde{I}_{p}} t\right)^{-(l+j-k)} \log (1+t / \mu) d t}_{\Xi_{0}}) \\
& -\underbrace{\sum_{\tau=2}^{l+j-k} \frac{(-1)^{l-k}}{\tau-1}\left(\tilde{I}_{p}\right)^{l-k-(\tau-1)}\left(\tilde{I}_{p}-\beta_{h} \tilde{P}_{I_{i}} \tilde{\alpha}_{f, i} \mu\right)^{-(l+j-k-\tau+1)}}_{\Xi_{3}} d t
\end{aligned}
$$

$$
\begin{aligned}
& \Xi_{2}=\sum_{v_{1}=1}^{l}\left(\begin{array}{c}
\Delta_{2,1}-1 \\
l-v_{1}
\end{array}\right)\left(-\frac{\beta_{h} \tilde{P}_{I_{i}} \tilde{\alpha}_{f, i}}{\beta_{h} \tilde{P}_{I_{i}} \tilde{\alpha}_{f, i} \mu-\tilde{I}_{p}}\right)^{l-v_{1}}\left(1-\frac{\beta_{h} \tilde{P}_{I_{i}} \tilde{\alpha}_{f, i} \mu}{\beta_{h} \tilde{P}_{I_{i}} \tilde{\alpha}_{f, i} \mu-\tilde{I}_{p}}\right)^{-\Delta_{2,1}} \frac{\mu^{-v_{1}}}{\Gamma\left(v_{1}\right)}\left(\tilde{\beta}_{g}\right)^{-(p+1)}
\end{aligned}
$$

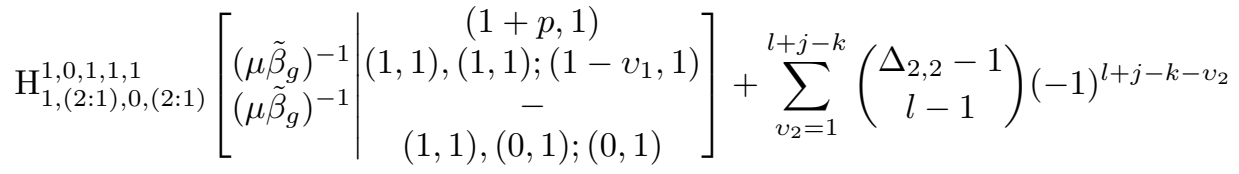

$$
\begin{aligned}
& \left(\frac{\tilde{I}_{p}}{\beta_{h} \tilde{P}_{I_{i}} \tilde{\alpha}_{f, i}}\right)^{-\Delta_{2,2}} \frac{\left(\tilde{\beta}_{g}\right)^{-(p+1)}}{\Gamma\left(v_{2}\right)} \mathrm{H}_{1,(2: 1), 0,(2: 1)}^{1,0,1,1}\left[\begin{array}{c|c}
\left(\mu \tilde{\beta}_{g}\right)^{-1} & (1+p, 1) \\
\frac{\beta_{h} \tilde{P}_{I^{\prime}} \tilde{\alpha}_{f, i}}{\left(\beta_{h} \tilde{P}_{I_{i}} \tilde{\alpha}_{f, i} \mu-\tilde{I}_{p}\right) \tilde{\beta}_{g}} & (1,1),(1,1) ;\left(1-v_{2}, 1\right) \\
- & (1,1),(0,1) ;(0,1)
\end{array}\right] .
\end{aligned}
$$

$$
\Xi_{3}=\frac{\mu^{-l}\left(\tilde{\beta}_{g}\right)^{-(p+1)}}{\Gamma(l) \Gamma(l+j-k-\tau+1)} \mathrm{H}_{1,(1: 1), 0,(1: 1)}^{1,1,1,1)}\left[\begin{array}{c|c}
\left(\mu \tilde{\beta}_{g}\right)^{-1} & (1+p, 1) \\
\frac{\beta_{h} \tilde{P}_{I_{i}} \tilde{\alpha}_{f, i}}{\left(\beta_{h} \tilde{P}_{I_{i}} \tilde{\alpha}_{f, i} \mu-\tilde{I}_{p}\right) \tilde{\beta}_{g}} & (1-l, 1) ;(k+\tau-l-j, 1) \\
- & (0,1) ;(0,1)
\end{array}\right] .
$$

calculate $\Lambda_{1}$ as

$$
\begin{aligned}
\Lambda_{1}= & \sum_{i=0}^{\tilde{l}-1}\left(\begin{array}{c}
\tilde{l}-1 \\
i
\end{array}\right) \mu^{\tilde{l}-1-i}\left[\frac{\Gamma(i+1)}{\left(\tilde{\beta}_{g}\right)^{i+1}} \log (\mu)+\right. \\
& \left.\left(\tilde{\beta}_{g}\right)^{-i-1} \int_{0}^{\infty} t^{i} e^{-t} \log \left(\frac{t}{\mu \tilde{\beta}_{g}}+1\right) d t\right]
\end{aligned}
$$

which is equivalent to the following expression:

$$
\begin{aligned}
\Lambda_{1}= & \sum_{i=0}^{\tilde{l}-1}\left(\begin{array}{c}
\tilde{l}-1 \\
i
\end{array}\right) \frac{\mu^{\tilde{l}-1-i}}{\left(\tilde{\beta}_{g}\right)^{i+1}}[\Gamma(i+1) \log (\mu)+ \\
& \sum_{j=0}^{i} \frac{\Gamma(i+1)}{\Gamma(i-j+1)}\left((-1)^{i-j-1}\left(\mu \tilde{\beta}_{g}\right)^{i-j} e^{\mu \tilde{\beta}_{g}} \operatorname{Ei}\left(-\mu \tilde{\beta}_{g}\right)+\right. \\
& \left.\left.\sum_{k=1}^{i-j} \Gamma(k)\left(-\mu \tilde{\beta}_{g}\right)^{i-j-k}\right)\right] .
\end{aligned}
$$

Similarly, we can obtain $\Lambda_{2}$ as

$$
\begin{aligned}
\Lambda_{2}= & \sum_{i=0}^{\tilde{l}}\left(\begin{array}{l}
\tilde{l} \\
i
\end{array}\right) \frac{\mu^{\tilde{l}-i}}{\left(\tilde{\beta}_{g}\right)^{i+1}}[\Gamma(i+1) \log (\mu)+ \\
& \sum_{j=0}^{i} \frac{\Gamma(i+1)}{\Gamma(i-j+1)}\left((-1)^{i-j-1}\left(\mu \tilde{\beta}_{g}\right)^{i-j} e^{\mu \tilde{\beta}_{g}} \operatorname{Ei}\left(-\mu \tilde{\beta}_{g}\right)+\right. \\
& \left.\left.\sum_{k=1}^{i-j} \Gamma(k)\left(-\mu \tilde{\beta}_{g}\right)^{i-j-k}\right)\right] .
\end{aligned}
$$

Substituting (E.7) into (E.6), we have

$$
\Theta_{2}=\Theta_{1}\left(1-F_{X}(\mu)\right)+\widetilde{\sum} e^{-\tilde{\beta}_{g} \mu}\left(\tilde{l} \Lambda_{1}-\tilde{\beta}_{g} \Lambda_{2}\right) .
$$

Thus, combining (E.11) and (E.1), we obtain (19). 


$$
\begin{aligned}
& \zeta=\left(-\tilde{I}_{p}\right)^{l-k}\left(\tilde{I}_{p}-\beta_{h} \tilde{P}_{I_{i}} \tilde{\alpha}_{f, i} \mu\right)^{-(l+j-k)}\left(\log \left(\frac{\tilde{I}_{p}}{\beta_{h} \tilde{P}_{I_{i}} \tilde{\alpha}_{f, i} \mu}\right) \mu^{-l}\left(\tilde{\beta}_{g}\right)^{-(p+1)} \Gamma(1+p)\right. \\
& \mathrm{F}_{0: 0}^{1: 1}\left[\begin{array}{cccccc}
1+p & : & l & ; l+j-k & ; & -\left(\mu \tilde{\beta}_{g}\right)^{-1},-\frac{\beta_{h} \tilde{P}_{I_{i}} \tilde{\alpha}_{f, i}}{\left(\beta_{h} \tilde{P}_{I_{i}} \tilde{\alpha}_{f, i} \mu-\tilde{I}_{p}\right) \tilde{\beta}_{g}}
\end{array}\right]- \\
& \sum_{v_{1}=1}^{l}\left(\begin{array}{c}
\Delta_{2,1}-1 \\
l-v_{1}
\end{array}\right)\left(-\frac{\beta_{h} \tilde{P}_{I_{i}} \tilde{\alpha}_{f, i}}{\beta_{h} \tilde{P}_{I_{i}} \tilde{\alpha}_{f, i} \mu-\tilde{I}_{p}}\right)^{l-v_{1}}\left(1-\frac{\beta_{h} \tilde{P}_{I_{i}} \tilde{\alpha}_{f, i} \mu}{\beta_{h} \tilde{P}_{I_{i}} \tilde{\alpha}_{f, i} \mu-\tilde{I}_{p}}\right)^{-\left(\Delta_{2,1}\right)} \frac{\mu^{-v_{1}}}{\Gamma\left(v_{1}\right)}\left(\tilde{\beta}_{g}\right)^{-(p+1)}
\end{aligned}
$$

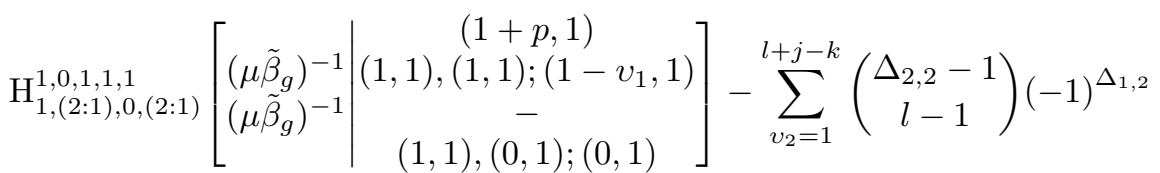

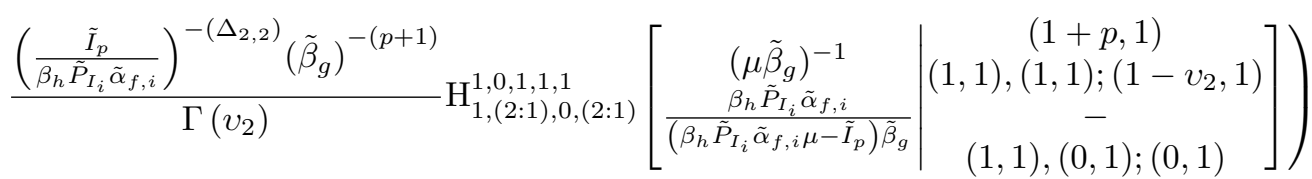

$$
\begin{aligned}
& -\sum_{\tau=2}^{l+j-k} \frac{(-1)^{l-k}}{\tau-1}\left(\tilde{I}_{p}\right)^{l-k-(\tau-1)}\left(\tilde{I}_{p}-\beta_{h} \tilde{P}_{I_{i}} \tilde{\alpha}_{f, i} \mu\right)^{-(l+j-k-\tau+1)} \mu^{-l}\left(\tilde{\beta}_{g}\right)^{-(p+1)} \Gamma(1+p)
\end{aligned}
$$

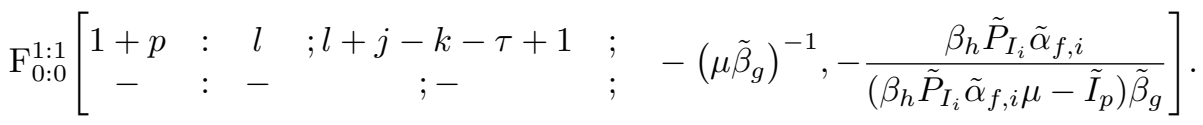

$$
\begin{aligned}
\Theta_{1}= & \sum_{l=1}^{L_{1}-1} \frac{\left(\beta_{h}\right)^{l} l}{\Gamma(l+1)} \sum_{i=1}^{M} \sum_{j=1}^{L_{2}} \frac{(-1)^{j} \theta_{i, j}}{\Gamma(j)} \int_{0}^{\infty} e^{-\frac{t}{\bar{P}_{I_{i}} \tilde{\alpha}_{f, i}}}(t)^{l+j-1} \int_{0}^{\infty} e^{-t \beta_{h} x} x^{l-1} \log (x) d x d t- \\
& \sum_{l=0}^{L_{1}-1} \frac{\left(\beta_{h}\right)^{l+1}}{\Gamma(l+1)} \sum_{i=1}^{M} \sum_{j=1}^{L_{2}} \frac{(-1)^{j} \theta_{i, j}}{\Gamma(j)} \int_{0}^{\infty} e^{-\frac{t}{\tilde{P}_{i} \tilde{\alpha}_{f, i}}}(t)^{l+j} \int_{0}^{\infty} e^{-t \beta_{h} x} x^{l} \log (x) d x d t \\
= & \sum_{l=1}^{L_{1}-1} \sum_{i=1}^{M} \sum_{j=1}^{L_{2}} \frac{(-1)^{j} \theta_{i, j} l \Gamma(l)\left(\tilde{P}_{I_{i}} \tilde{\alpha}_{f, i}\right)^{j}}{\Gamma(l+1)}\left[\psi(l)-\log \left(\beta_{h}\right)-\psi(j)-\log \left(\tilde{P}_{I_{i}} \tilde{\alpha}_{f, i}\right)\right]- \\
& \sum_{l=0}^{L_{1}-1} \sum_{i=1}^{M} \sum_{j=1}^{L_{2}}(-1)^{j} \theta_{i, j}\left(\tilde{P}_{I_{i}} \tilde{\alpha}_{f, i}\right)^{j}\left[\psi(l+1)-\log \left(\beta_{h}\right)-\psi(j)-\log \left(\tilde{P}_{I_{i}} \tilde{\alpha}_{f, i}\right)\right] .
\end{aligned}
$$

$$
\begin{aligned}
\Lambda & =\widetilde{\sum}\left[\tilde{l} \int_{\mu}^{\infty} t^{\tilde{l}-1} e^{-\tilde{\beta}_{g} t} \log (t) d t-\tilde{\beta}_{g} \int_{\mu}^{\infty} t^{\tilde{l}} e^{-\tilde{\beta}_{g} t} \log (t) d t\right] \\
& =\widetilde{\sum} e^{-\tilde{\beta}_{g} \mu}[\tilde{l} \underbrace{\int_{0}^{\infty}(t+\mu)^{\tilde{l}-1} e^{-\tilde{\beta}_{g} t}\left(\log \left(\frac{t}{\mu}+1\right)+\log (\mu)\right) d t}_{\Lambda_{1}}-\tilde{\beta}_{g} \underbrace{\int_{0}^{\infty}(t+\mu)^{\tilde{l}} e^{-\tilde{\beta}_{g} t}\left(\log \left(\frac{t}{\mu}+1\right)+\log (\mu)\right) d t}_{\Lambda_{2}}] .
\end{aligned}
$$




\section{REFERENCES}

[1] D. Chen, S. Yin, Q. Zhang, M. Liu, and S. Li, "Mining spectrum usage data: A large-scale spectrum measurement study," in Proc. ACM Int. Conf. on Mobile Computing and Networking, Beijing, China, Sep. 2009, pp. 13-24.

[2] M. Wellens, J. Wu, and P. Mahonen, "Evaluation of spectrum occupancy in indoor and outdoor scenario in the context of cognitive radio," in Proc. Int. Conf. on Cognitive Radio Oriented Wireless Networks, Orlando, FL, Jul. 2007, pp. 420-427.

[3] M. Lopez-Benitez, A. Umbert, and F. Casadevall, "Evaluation of spectrum occupancy in Spain for cognitive radio applications," in Proc. IEEE Veh. Techno. Conf. Spring, Barcelona, Spain, Apr. 2009, pp. 1-5.

[4] K. A. Qaraqe, H. Celebi, A. Gorcin, A. El-Saigh, H. Arslan, and M.S. Alouini, "Empirical results for wideband multidimensional spectrum usage," in Proc. IEEE Int. Symp. on Personal, Indoor and Mobile Radio Commun., Tokyo, Japan, Sep. 2009, pp. 1262-1266.

[5] M. H. Islam, C. L. Koh, S. W. Oh, X. Qing, Y. Y. Lai, C. Wang, Y.-C. Liang, B. E. Toh, F. Chin, G. L. Tan, and W. Toh, "Spectrum survey in Singapore: Occupancy measurements and analyses," in Proc. Int. Conf. on Cognitive Radio Oriented Wireless Networks, Singapore, May 2008, pp. 1-7.

[6] M. A. McHenry, "NSF spectrum occupancy measurements project summary," Shared Spectrum Co., Tech. Rep., Aug. 2005.

[7] V. N. Q. Bao, L. Q. Cuong, L. Q. Phu, T. D. Thuan, L. M. Trung, and N. T. Quy, "Spectrum survey in Vietnam: Occupancy measurements and analysis for cognitive radio applications," in Proc. Advanced Techno. for Commun., Da Nang, Vietnam, Aug. 2011, pp. 135-143.

[8] J. Mitola and G. Q. Maguire, Jr., "Cognitive radio: making software radio more personal," IEEE Pers. Commun., vol. 6, no. 4, pp. 13-18, Aug. 1999.

[9] E. Biglieri, A. Goldsmith, L. J. Greenstein, N. B. Mandayam, and H. V. Poor, Principles of Cognitive Radio. Cambridge, UK: Cambridge University Press, 2013.

[10] A. Ghasemi and E. S. Sousa, "Fundamental limits of spectrum-sharing in fading environments," IEEE Trans. Wireless Commun., vol. 6, no. 2, pp. 649-658, Feb. 2007.

[11] H. A. Suraweera, P. J. Smith, and M. Shafi, "Capacity limits and performance analysis of cognitive radio with imperfect channel knowledge," IEEE Trans. Veh. Technol., vol. 59, no. 4, pp. 1811-1822, May 2010.

[12] C. Zhong, T. Ratnarajah, and K.-K. Wong, "Outage analysis of decodeand-forward cognitive dual-hop systems with the interference constraint in Nakagami-m fading channels," IEEE Trans. Veh. Technol., vol. 60 , pp. 2875-2879, Jul. 2011.

[13] T. W. Ban, W. Choi, B. C. Jung, and D. K. Sung, "Multi-user diversity in a spectrum sharing system," IEEE Trans. Wireless Commun., vol. 8, no. 1, pp. 102-106, Jan. 2009.

[14] K. J. Kim, T. Q. Duong, H. V. Poor, and L. Shu, "Performance analysis of cyclic prefixed single-carrier spectrum sharing relay systems in primary user interference," IEEE Trans. Signal Process., vol. 60, no. 12, pp. 6729-6734, Dec. 2012.

[15] K. J. Kim, T. Q. Duong, and X.-N. Tran, "Performance analysis of cognitive spectrum-sharing single-carrier systems with relay selection," IEEE Trans. Signal Process., vol. 60, no. 12, pp. 6435-6449, Dec. 2012.

[16] K. J. Kim, T. Q. Duong, and H. V. Poor, "Outage probability of singlecarrier cooperative spectrum sharing systems with decode-and-forward relaying and selection combining," IEEE Trans. Wireless Commun., vol. 12 , no. 2 , pp. $806-817$, Feb. 2013.

[17] N. Al-Dhahir, "Single carrier frequency domain equalization for space time block-coded transmissions over frequency-selective fading channels," IEEE Commun. Lett., vol. 7, no. 11, pp. 304-306, Jul. 2001.

[18] H. Mheidat, M. Uysal, and N. Al-Dhahir, "Equalization techniques for distributed space-time block codes with amplify-and-forward relaying," IEEE Trans. Signal Process., vol. 55, no. 5, pp. 1839-1852, May 2007.

[19] D.-Y. Seol, U.-K. Kwon, G.-H. Im, and E.-S. Kim, "Relay-based single carrier transmission with SFBC in uplink fast fading channels," IEEE Commun. Lett., vol. 12, no. 12, pp. 928-930, Dec. 2007.
[20] K. J. Kim, Y. Li, and T. A. Tsiftsis, "On the performance of cyclicprefixed single-carrier cellular systems in cochannel interference," IEEE Trans. Veh. Technol., vol. 60, no. 8, pp. 4035-4040, Oct. 2011.

[21] T. Q. Duong, P. L. Yeoh, V. N. Q. Bao, M. Elkashlan, and N. Yang, "Cognitive relay networks with multiple primary transceivers under spectrum-sharing," IEEE Signal Process. Lett., vol. 19, no. 11, pp. 741744, Nov. 2012.

[22] K. J. Kim, T. Q. Duong, M. Elkashlan, P. L. Yeoh, H. V. Poor, and M. H. Lee, "Spectrum sharing single-carrier in the presence of multiple licensed receivers," IEEE Trans. Wireless Commun., vol. 12, no. 10, pp. 5223-5235, Oct. 2013.

[23] Y. Zou, J. Zhu, B. Zheng, and Y.-D. Yao, "An adaptive cooperating diversity scheme with best-relay selection in cognitive radio networks," IEEE Trans. Signal Process., vol. 58, no. 10, pp. 5438-5445, Oct. 2010.

[24] J. Lee, H. Wang, J. G. Andrews, and D. Hong, "Outage probability of cognitive relay networks with interference constraints," IEEE Trans. Wireless Commun., vol. 10, no. 2, pp. 390-395, Feb. 2011.

[25] H. Ding, J. Ge, D. da Costa, and Z. Jiang, "Asymptotic analysis of cooperative diversity systems with relay selection in a spectrum-sharing scenario," IEEE Trans. Veh. Technol., vol. 60, no. 2, pp. 457-472, Feb. 2011.

[26] H. Yu, I.-H. Lee, and G. L. Stuber, "Outage probability of decodeand-forward cooperative relaying systems with co-channel interference," IEEE Trans. Wireless Commun., vol. 11, no. 1, pp. 266-274, Jan. 2011.

[27] P. A. Dmochowski, H. A. Suraweera, P. J. Smith, and M. Shafi, "Impact of channel knowledge on cognitive radio system capacity," in Proc. IEEE Veh. Technol. Conf. (VTC 2010 Fall), Ottawa, Canada, Sep. 2010, pp. $1-5$.

[28] S. Kato, H. Harada, R. Funada, T. Baykas, C. S. Sum, J. Wang, and M. A. Rahman, "Single carrier transmission for multi-gigabit $60-\mathrm{GHz}$ WPAN systems," IEEE J. Sel. Areas Commun., vol. 27, no. 8, pp. 14661478, Oct. 2009.

[29] T.-H. Pham, Y.-C. Liang, A. Nallanathan, and H. Garg, "Optimal training sequences for channel estimation in bi-directional relay networks with multiple antennas," IEEE Trans. Commun., vol. 58, no. 2, pp. 474-479, Feb. 2010.

[30] P. R. Davis, Circulant Matrices. New York: John Wiley, 1979.

[31] K. J. Kim, T. A. Tsiftsis, and H. V. Poor, "Power allocation in cyclic prefixed single-carrier relaying systems," IEEE Trans. Wireless Commun., vol. 10, no. 7, pp. 2297-2305, Jul. 2011.

[32] T. Zhang, Y. Cai, and W. Yang, "Performance analysis of multi-source multi-relay wireless networks with co-channel interference," Electron. Lett., vol. 49, no. 4, pp. 304-305, Feb. 2013.

[33] I. S. Gradshteyn and I. M. Ryzhik, Table of Integrals, Series, and Products. New York: Academic Press, 2007.

[34] H. A. Suraweera, D. S. Michalopoulos, and C. Yuen, "Performance analysis of fixed gain relay systems with a single interferer in Nakagami$m$ fading channels," IEEE Trans. Veh. Technol., vol. 61, no. 3, pp. 102106, Mar. 2012

[35] H. Shin and J. B. Song, "MRC analysis of cooperative diversity with fixed-gain relays in Nakagami- $m$ fading channels," IEEE Trans. Wireless Commun., vol. 7, pp. 2069-2074, Jun. 2008.

[36] A. Lozano, A. M. Tulino, and S. Verdú, "High-SNR power offset in multiantenna communication," in Proc. IEEE Int. Symp. Inf. Theory, Chicago, IL, Jun. 2004, p. 287.

[37] X. W. Cui, Q. T. Zhang, and Z. M. Feng, "Outage performance for maximal ratio combiner in the presence of unequal-power co-channel interferers," IEEE Commun. Lett., vol. 8, no. 5, pp. 289-291, May 2004.

[38] A. M. Mathai and R. K. Saxena, The H-function with Applications in Statistics and Other Disciplines. New York: Wiley, 1978.

[39] A. P. Prudnikov, Y. A. Brychkov, , and O. I. Marichev, Integrals and Series. New York: Gordon and Breach Science, 1990. 


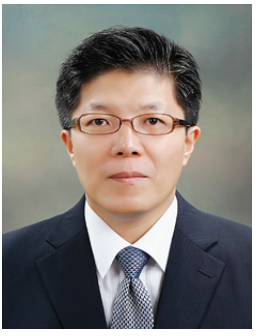

Kyeong Jin Kim (SM'11) received the M.S. degree from the Korea Advanced Institute of Science and Technology (KAIST) in 1991 and the M.S. and $\mathrm{Ph} . \mathrm{D}$. degrees in electrical and computer engineering from the University of California, Santa Barbara in 2000. During 1991-1995, he was a research engineer at the video research center of Daewoo Electronics, Ltd., Korea. In 1997, he joined the data transmission and networking laboratory, University of California, Santa Barbara. After receiving his degrees, he joined the Nokia research center (NRC) and Nokia Inc., Dallas, TX, as a senior research engineer, where he was, from 2005 to 2009 , an L1 specialist. During 2010-2011, he was an Invited Professor at Inha University, Korea. Since 2012, he has worked as a senior principal research staff member in the Mitsubishi Electric Research Laboratories (MERL), Cambridge, MA. His research has been focused on the transceiver design, resource management, scheduling in the cooperative wireless communications systems, cooperative spectrum sharing system, and device-to-device communications.

Dr. Kim currently serves as an editor for the IEEE COMMUNICATIONS LETTERS. He also serves as guest editors for the EURASIP JOURNAL ON Wireless COMmunications AND Networking: Special Issue on "Cooperative Cognitive Networks" and IET CommunicATIONS: Special Issue on "Secure Physical Layer Communications". He served as a TPC chair for the IEEE GLOBECOM 2013 and 2014 Workshop on Trusted Communications with Physical Layer Security.

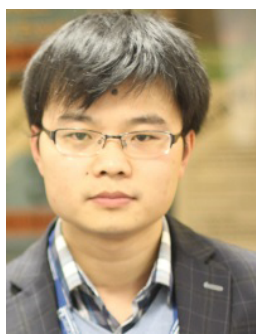

Lifeng Wang (S'12) is working towards his Ph.D. degree in Electronic Engineering at Queen Mary University of London. Before that, he received the M.S. degree in Electronic Engineering from the University of Electronic Science and Technology of China, in 2012. His research interests include MIMO, cooperative communications, cognitive radio, and physical layer security.

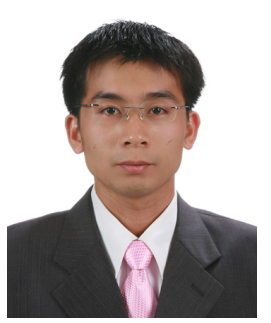

Trung Q. Duong (S'05, M'12, SM'13) received his Ph.D. degree in Telecommunications Systems from Blekinge Institute of Technology (BTH), Sweden in 2012, and then continued working at BTH as a project manager. Since 2013, he has joined Queen's University Belfast, UK as a Lecturer (Assistant Professor). He held a visiting position at Polytechnic Institute of New York University and Singapore University of Technology and Design in 2009 and 2011, respectively. His current research interests include cooperative communications, cognitive radio networks, physical layer security, massive MIMO, cross-layer design, mmwaves communications, and localization for radios and networks.

Dr. Duong has been a TPC chair for several IEEE international conferences and workshops including the most recently IEEE GLOBECOM13 Workshop on Trusted Communications with Physical Layer Security. He currently serves as an Editor for the IEEE COMMUNiCATIONS LETTERS, Wiley TRANSACTIONS ON EMERGING TELECOMMUNICATIONS TECHNOLOGIES. Dr. Duong has served as the Lead Guest Editor of the special issue on "Location Awareness for Radios and Networks" of the IEEE JOURNAL in Selected Areas on Communications, the Lead Guest Editor of the special issue on "Secure Physical Layer Communications" of the IET Communications, Guest Editor of the special issue on "Green Media: Toward Bringing the Gap between Wireless and Visual Networks" of the IEEE WIRELESS COMMUNICATIONS MAGAZINE, Guest Editor of the special issue on "Millimeter Wave Communications for 5G" and "Energy Harvesting Communications" of the IEEE COMmUniCATIONS MAGAZINE, Guest Editor of the special issue on "Cooperative Cognitive Networks" of the EURASIP Journal ON Wireless COMMUNiCATIONS AND NETWORKING, Guest Editor of special issue on "Security Challenges and Issues in Cognitive Radio Networks" of the EURASIP JoURnal on AdVANCES Signal ProcessING. He is awarded the Best Paper Award at the IEEE Vehicular Technology Conference (VTC-Spring) in 2013 and the Exemplary Reviewer Certificate of the IEEE Communications Letters in 2012.

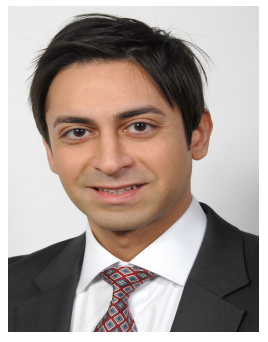

Maged Elkashlan (M'06) received the Ph.D. degree in Electrical Engineering from the University of British Columbia, Canada, 2006. From 2006 to 2007, he was with the Laboratory for Advanced Networking at University of British Columbia. From 2007 to 2011, he was with the Wireless and Networking Technologies Laboratory at Commonwealth Scientific and Industrial Research Organization (CSIRO), Australia. During this time, he held an adjunct appointment at University of Technology Sydney, Australia. In 2011, he joined the School of Electronic Engineering and Computer Science at Queen Mary University of London, UK, as an Assistant Professor. He also holds visiting faculty appointments at the University of New South Wales, Australia, and Beijing University of Posts and Telecommunications, China. His research interests fall into the broad areas of communication theory, wireless communications, and statistical signal processing for distributed data processing, millimeter wave communications, cognitive radio, and wireless security.

Dr. Elkashlan currently serves as an Editor of the IEEE TRANSACTIONS ON WIRELESS COMMUNICATIONS, the IEEE TRANSACTIONS ON VEHICULAR TECHNOLOGY, and the IEEE COMMUNiCATIONS LETTERS. He also serves as the Lead Guest Editor for the special issue on "Green Media: The Future of Wireless Multimedia Networks" of the IEEE WIRELESS COMMUNICATIONS MAGAZINE, Lead Guest Editor for the special issue on "Millimeter Wave Communications for 5G" of the IEEE COMMUNICATIONS MAGAZINE, Guest Editor for the special issue on "Energy Harvesting Communications" of the IEEE COMMUNICATIONS MAGAZINE, and Guest Editor for the special issue on "Location Awareness for Radios and Networks" of the IEEE JOURNAL on Selected Areas in Communications. He received the Best Paper Award at the IEEE Vehicular Technology Conference (VTC-Spring) in 2013. He received the Exemplary Reviewer Certificate of the IEEE Communications Letters in 2012.

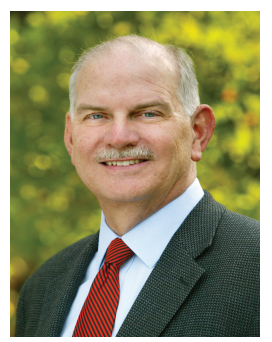

H. Vincent Poor (S'72, M'77, SM'82, F'87) received the Ph.D. degree in EECS from Princeton University in 1977. From 1977 until 1990, he was on the faculty of the University of Illinois at UrbanaChampaign. Since 1990 he has been on the faculty at Princeton, where he is the Michael Henry Strater University Professor of Electrical Engineering and Dean of the School of Engineering and Applied Science. Dr. Poor's research interests are in the areas of information theory, statistical signal processing and stochastic analysis, and their applications in wireless networks and related fields including social networks and smart grid. Among his publications in these areas are the recent books Principles of Cognitive Radio (Cambridge University Press, 2013) and Mechanisms and Games for Dynamic Spectrum Allocation (Cambridge University Press, 2014).

Dr. Poor is a member of the National Academy of Engineering and the National Academy of Sciences, and a foreign member of Academia Europaea and the Royal Society. He is also a fellow of the American Academy of Arts and Sciences, the Royal Academy of Engineering (U.K.), and the Royal Society of Edinburgh. He received the Marconi and Armstrong Awards of the IEEE Communications Society in 2007 and 2009, respectively. Recent recognition of his work includes the 2011 IEEE Eric E. Sumner Award, the 2014 URSI Booker Gold Medal, and honorary doctorates from Aalborg University, the Hong Kong University of Science and Technology, and the University of Edinburgh. 